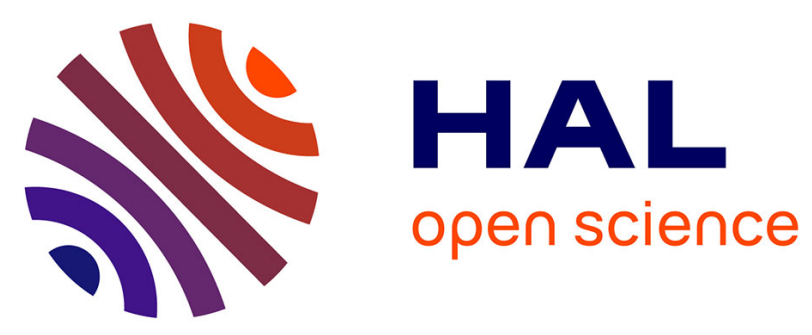

\title{
The formulation of dynamical contact problems with friction in the case of systems of rigid bodies and general discrete mechanical systems-Painlevé and Kane paradoxes revisited
}

Alexandre Charles, Patrick Ballard

\section{To cite this version:}

Alexandre Charles, Patrick Ballard. The formulation of dynamical contact problems with friction in the case of systems of rigid bodies and general discrete mechanical systems-Painlevé and Kane paradoxes revisited. Zeitschrift für Angewandte Mathematik und Physik, 2016, 67 (4), 10.1007/s00033016-0688-1 . hal-02326946

\section{HAL Id: hal-02326946 \\ https://hal.science/hal-02326946}

Submitted on 22 Oct 2019

HAL is a multi-disciplinary open access archive for the deposit and dissemination of scientific research documents, whether they are published or not. The documents may come from teaching and research institutions in France or abroad, or from public or private research centers.
L'archive ouverte pluridisciplinaire HAL, est destinée au dépôt et à la diffusion de documents scientifiques de niveau recherche, publiés ou non, émanant des établissements d'enseignement et de recherche français ou étrangers, des laboratoires publics ou privés. 


\title{
The formulation of dynamical contact problems with friction in the case of systems of rigid bodies and general discrete mechanical systems - Painlevé and Kane paradoxes revisited
}

\author{
Alexandre Charles and Patrick Ballard
}

\begin{abstract}
The dynamics of mechanical systems with a finite number of degrees of freedom (discrete mechanical systems) is governed by the Lagrange equation which is a second-order differential equation on a Riemannian manifold (the configuration manifold). The handling of perfect (frictionless) unilateral constraints in this framework (that of Lagrange's analytical dynamics) was undertaken by Schatzman and Moreau at the beginning of the 1980s. A mathematically sound and consistent evolution problem was obtained, paving the road for many subsequent theoretical investigations. In this general evolution problem, the only reaction force which is involved is a generalized reaction force, consistently with the virtual power philosophy of Lagrange. Surprisingly, such a general formulation was never derived in the case of frictional unilateral multibody dynamics. Instead, the paradigm of the Coulomb law applying to reaction forces in the real world is generally invoked. So far, this paradigm has only enabled to obtain a consistent evolution problem in only some very few specific examples and to suggest numerical algorithms to produce computational examples (numerical modelling). In particular, it is not clear what is the evolution problem underlying the computational examples. Moreover, some of the few specific cases in which this paradigm enables to write down a precise evolution problem are known to show paradoxes: the Painlevé paradox (indeterminacy) and the Kane paradox (increase of kinetic energy due to friction). In this paper, we follow Lagrange's philosophy and formulate the frictional unilateral multibody dynamics in terms of the generalized reaction force and not in terms of the real world reaction force. A general evolution problem that governs the dynamics is obtained for the first time. We prove that all the solutions are dissipative, that is, this new formulation is free of Kane paradox. We also prove that some indeterminacy of the Painlevé paradox is fixed in this formulation.
\end{abstract}

Mathematics Subject Classification (2010). 70F35, 70F40.

Keywords. Contact, dry friction, analytical dynamics, Cauchy problem, formulation.

\section{Introduction}

A large number of engineering problems are still conveniently studied in the general framework of the dynamics of rigid body systems. A lot of common applications, such as the computation of the dynamics of granular media in civil engineering or the analysis of a mechanized arm grabbing an object in robotics, require the handling of non-penetration conditions (synonymously contact conditions) and/or friction within the general theory of rigid body dynamics.

Until the late 1970s, the usual practice in rigid body dynamics with contact and friction was to write a collection of ordinary differential equations. There was a differential equation governing the situation where body 1 achieves active contact with body 2 , a differential equation governing the 
situation where body 1 achieves active contact with body 3, etc... One was supposed to switch from one equation to another according to the situation which was met. This point of view is referred to as event-driven (this terminology seems to be due to Jean Jacques Moreau) in this paper, but the denomination of hybrid systems is also frequently encountered.

The event-driven point of view is convenient neither for theoretical investigations nor for computational purposes (especially in situations where infinitely many impacts accumulate in a bounded time interval). A new strategy emerged at the end of the 1970s. It was originally developed by Michelle Schatzman [25] who was the first to formulate a global (with respect to time) evolution problem governing the frictionless bouncing of a point particle against some obstacle. Her formulation was the first one to encompass the episodes of smooth motion as well as the impacts possibly encountered during the evolution of the system. The price to pay was that the problem was no longer governed by an ordinary differential equation, but by something more complicated involving extensive use of measure theory (in this framework, the percussion, that is the instantaneous reaction force exerted by the obstacle during a collision is represented by a Dirac measure with respect to time). As an immediate outcome of such a formulation, she was able to implement a penalty technique to prove the existence of a solution. The setting of Michelle Schatzman [25] was restricted to the (frictionless) dynamical motion of a point particle (or a finite collection of such points). It was rapidly extended by Jean Jacques Moreau [15] to the situation of the dynamic motion of a finite collection of rigid bodies satisfying frictionless noninterpenetration conditions and possibly bouncing against finitely many (frictionless) fixed external obstacles. The formulation of Jean Jacques Moreau was a suitable modification of the classical Lagrange equations governing the dynamic motion of a finite collection of rigid bodies, in which Lagrange generalized acceleration was allowed to be a measure (with respect to time) to account for the (generalized) velocity discontinuities occuring during collisions, in the line of the setting of Michelle Schatzman for point particles. In particular, the reaction force involved in Moreau's formulation of frictionless unilateral dynamics is a generalized reaction force, consistently with the framework of Lagrange. In some sense, the article [15] of Jean Jacques Moreau has made clear how to formulate consistently the frictionless unilateral dynamics of a finite collection of rigid bodies with enough generality to encompass any practical situation raised by engineering applications. This opened the way to numerous theoretical investigations on frictionless multibody dynamics. Around 1985, Monteiro Marques proved the first convergence results for the time-stepping technique introduced by Moreau for the sake of numerical computation (see the monograph [14] and the references to the original articles therein). In Monteiro Marques's approach, the time-stepping was another technique, alternative to Michelle Schatzman's penalty method, to prove the existence of a solution. It had the advantage of being directly related to a strategy to produce numerical approximations. In [14], Monteiro Marques's existence proof was restricted to the case of the completely inelastic bouncing of a point particle against one smooth obstacle. Then, subsequent refinements of the time-stepping technique were worked out by Michelle Schatzman, Manuel Monteiro Marques, Laetitia Paoli and coworkers to prove always more general existence results applying to more general impact laws, rigid solids instead of point particles, several unilateral constraints instead of only one... A recent account of this line of research which is also relevant from the point of view of analysing numerical strategies, can be found in $[20,21]$. In parallel of these investigations on the convergence of time-stepping schemes and the existence of solution of the evolution problem formulated by Moreau, the question of uniqueness of solution was also studied, motivated by a striking example contained in Schatzman's original paper [25], exhibiting two distinct solutions in an evolution problem with all data being of class $C^{\infty}$. Danilo Percivale $[23,24]$ was the first to suggest a possible workaround by requiring all the data to be analytic instead of only $C^{\infty}$. But, he was only able to prove uniqueness of solution in very restrictive situations. This was generalized by Ballard [1,3] who proved the existence and uniqueness of a solution for the most general evolution problem raised by frictionless unilateral multibody dynamics, as formulated by Moreau in the most general case (multi-constraints, arbitrary impact law, arbitrary mass matrix), but under the regularity requirement of analyticity. Hence, it can be said that frictionless unilateral multibody dynamics has reached today a certain stage of completion involving : 
- a sound and consistent mathematical formulation of the general evolution problem, encompassing most of the situations faced in engineering applications,

- a general existence and uniqueness theory for that evolution problem,

- convergence proof for the main numerical algorithms designed to compute approximate solutions.

Naturally, there is still matter for theoretical investigations in that field. A stability theory, similar to that which is known in smooth mechanics, is still to be developed in this framework. The same is true of a theory of control in frictionless unilateral multibody. However, no doubt that any future theoretical investigations in these fields will strongly rely on the general formulation of the evolution problem and its analysis.

Meanwhile, in most use of unilateral multibody dynamics in engineering problems, it is desirable to account for friction. Unfortunately, frictional unilateral multibody dynamics has not reached yet the same stage of completion as in the frictionless case. Actually, the case of a collection of point particles must be distinguished from the case of a collection of rigid bodies.

- In the case of a collection of point particles, Moreau [16] and Monteiro Marques [14] worked out a consistent formulation of the evolution problem that implement Coulomb friction. Monteiro Marques proved the existence of a solution (case of a single constraint and completely inelastic impact law) by proving the convergence of a sequence of approximations built by use of an appropriate time-stepping method. Recently, Charles and Ballard [5] proved the existence, but also the uniqueness of a solution under the additional regularity hypothesis of analyticity of the data, for the most general evolution problem raised by the frictional unilateral dynamics of a finite collection of point particles (case of several constraints and an arbitrary impact law). Their evolution problem encompasses that of Moreau and Monteiro Marques as a particular case.

- In the case of a collection of rigid bodies, the situation is rather different and, surprisingly no general formulation of the dynamics can be found in the literature. By general formulation, we simply mean a precise set of mathematical conditions to be checked to decide whether a given motion of the system is an admissible dynamical evolution or not, as is available for the frictionless multibody dynamics and that will be recalled in this article (problem $\mathcal{P}_{2}$ ). Instead of such a precise formulation, the general idea that prevails in the field is that the dynamics is governed by a dynamical equation involving reaction forces, complemented by suitable contact conditions and friction law. But, the usual laws for dry friction (such as the Coulomb law) are commonly formulated in terms of reaction forces in the real world, that is, forces which are represented as vectors in the usual three-dimensional space, and in terms of the velocities of the material contact points. This paradigm has not led so far to any general formulation of frictional multibody dynamics. It has only resulted in two outcomes:

- A consistent formulation of the frictional dynamics in very specific cases, such as the Painlevé example. Such a consistent formulation (based on the paradigm of Coulomb law applying to reaction forces of the real world) for the case of only one material contact point (which contains the Painlevé example) and the completely inelastic impact law can be found in Stewart [26]. The motivation of Stewart was to prove the existence of a dynamical solution for the Painlevé example, since suspicion of non-existence of solution had fed a one century old controversy which could be entitled: "is the (local) Coulomb law consistent with rigid body dynamics?'.

- Numerical methods to compute discrete-time solutions in more complex cases. Examples of such methods are those of Jean [9], which was inspired by the time-stepping technique of Monteiro Marques, and the method of Stewart in [26]. A common feature of all these methods is that they are derived on the basis of the paradigm of the Coulomb law applying to forces of the real world. But, they are not derived on the basis of a general continuous-time evolution problem. This was recognized by Stewart in [27]: 'In many ways it is easier to write down a numerical method for rigid-body dynamics than it is to say exactly what the method is trying to compute.' 
The formulation of Stewart (based on the paradigm of Coulomb law applying to reaction forces of the real world) for the plane problem and only one material point will be discussed in section 6 of this paper. It is the formulation that underlies the Painlevé example. Stewart proved in [26] the existence of a solution in the restrictive case where the impact law is the completely inelastic one. In that case, it is well-known that there may be multiple solutions for this Cauchy problem. This indeterminacy is what we address as Painlevé paradox in this paper. Stewart also proved in [26] that the particular solution he constructs (we recall that there may exist multiple solutions in general) is dissipative, that is, the sum of the kinetic energy and the potential energy is not increasing with time. This is important because Kane [10] exhibited in 1983 a striking example that falls into Stewart's general framework (except that the restitution coefficient is not zero in Kane's example), and where certain situations of collision necessarily increase the kinetic energy of the system. This possible increase of kinetic energy is what we address as Kane paradox in this paper. Kane's example proves that the dissipativity result in [26] cannot extend to the situation of a restitution coefficient of 0.5 .

To summarize, the paradigm of Coulomb law applying to reaction forces of the real world has not resulted yet in any precise formulation of a general continuous-time evolution problem. In addition, in the few specific cases where such a precise evolution problem was obtained, it turned out to show indeterminacy (multiple solutions for the Cauchy problem) and energetic inconsistency (possible increase of kinetic energy during collisions). Writing down a sound and consistent formulation of a general continuous-time evolution problem may be felt not strictly necessary for the purpose of numerical modelling. However, it is an indispensable preliminary step for all the theoretical investigations such as the convergence analysis of a numerical method, or the development of a theory of stability or control. Deriving such a general formulation of frictional multibody dynamics is what this paper is about.

Our approach will rely on the following general prerequisites.

1. Mechanical consistency. In any theory of mechanics, the representation of forces must be consistent with the geometric modelling. For example, the Cauchy stress field is an appropriate representation of internal forces in a classical three-dimensional deformable continua, but speaking of the stress field inside a body which is modelled (idealized) as a rigid body is pointless. Modern mechanics has made this consistency systematic by requiring that forces should be dual of velocities through the principle of virtual powers (but this idea goes back to Lagrange). In a collection of rigid bodies (or, more precisely, of bodies which are modelled as rigid bodies), the appropriate representation of forces is the so-called Lagrange generalized force. In the case of a system of rigid bodies with contacts, one can always calculate the Lagrange generalized reaction force from the contact forces in the real world but, one cannot recover, in general, the contact forces distribution from a Lagrange generalized reaction force. Except in a few particular cases, the distribution of contact forces must be considered as indeterminate as the stress field is in each body. Here, the fact that the calculation of the contact force from the generalized reaction force is possible in a few specific examples such as the Painleve example, has maintained a confusion. In our opinion and in accordance with the modern point-of-view on mechanics systematized by the principle of virtual powers, a general consistent formulation of frictional multibody dynamics must be stated in terms of Lagrange generalized reaction force and not in terms of contact forces in the real world. Besides, this is the usual point of view when analysing the frictional relative motion of two flat rigid bodies as in Coulomb's original experiments [6] where the friction law is naturally expressed in terms of the total force (and the total force is precisely a component of the generalized force) and not in terms of the pointwise distribution of local contact forces which is undetermined in a framework of rigid bodies modelling.

2. Energetic consistency. Our choice is to invoke the principle of maximal dissipation as a fundamental axiom. This principle was already invoked in that context at least by Moreau in [18] and by Stewart in [26]. However, in these references, it was invoked in connection with a friction law formulated in terms of contact forces in the real world. On the opposite, we will invoke the principle of maximal dissipation in connection with the use Lagrange generalized forces to derive the flow rule of the friction law. 
We feel these prerequisites as basics and well accepted in the whole continuum mechanics. We are astounded that they have not been already applied to frictional unilateral multibody dynamics and our opinion is that this is the origin of the paradoxes of Kane and Painlevé. Once they are accepted, the derivation of a general evolution problem is straightforward. This general evolution problem is problem $\mathcal{P}_{4}^{\prime}$ in this paper. It encompasses the case of any discrete mechanical system with arbitrary mass matrix, an arbitrary number of unilateral constraint and the most general impact law. The friction law rules both the episodes of smooth motion and the collisions. Dissipativity of solutions for problem $\mathcal{P}_{4}^{\prime}$ is proved (proposition 5.1), that is, energetic consistency is built-in within problem $\mathcal{P}_{4}^{\prime}$ which is therefore free of the Kane paradox. In addition, it is proved that certain examples of indeterminacies as in the Painlevé example, are fixed in this new formulation. Showing that problem $\mathcal{P}_{4}^{\prime}$ is definitively free of indeterminacy would require a proof of existence and uniqueness of solution. Such a result is highly plausible under the additional assumption of analyticity of the data since it was already proved for problem $\mathcal{P}_{4}$ in [5], and that problem $\mathcal{P}_{4}^{\prime}$ has a similar structure. The main difference is that one may have unbounded friction sets in problem $\mathcal{P}_{4}^{\prime}$. Proving the existence and uniqueness of solutions of problem $\mathcal{P}_{4}^{\prime}$ along the same line as in [5], and thus, ruling out rigorously the indeterminacy of Painlevé paradox for problem $\mathcal{P}_{4}^{\prime}$ is postponed to future work.

The article is organized as follows. Section 1 draws a general picture of the Lagrangian formulation of the dynamics of a collection of rigid bodies and sketches how (perfect) bilateral constraints are handled in this framework as well as frictionless unilateral constraints in the lines of Moreau [15] and Ballard [3]. The general idea is that the evolution problem for the dynamics of a system of rigid bodies is the same as that for a point particle except that the point evolves in a space of dimension $d$ instead of the usual three-dimensional space and that we have a mass matrix that depends on the current configuration $\mathbf{q}$, in general. In section 2 , the simple situation of the dynamics of a point particle in frictional contact with one smooth obstacle is considered since this is a situation in which everybody agrees on how the problem should be formulated. This problem is exactly the one for which Monteiro Marques [14] proved the existence of a solution by use of a time-stepping technique. The fact that uniqueness of solution also holds true in the analytic case is proved in [5]. In section 3, we extend the previous formulation to the situation of an arbitrary discrete system (such as a rigid body, for example) with one frictional constraint in the spirit of Lagrange's point of view on rigid body dynamics. The generalization of the previous formulation to the case of several constraints is obtained first in the case of a point particle first (section 4) and in the case of an arbitrary collection of rigid bodies then (section 5). This analysis results in problem $\mathcal{P}_{4}^{\prime}$ which is the general evolution problem governing the dynamics of an arbitrary discrete mechanical system with several unilateral constraints. In problem $\mathcal{P}_{4}^{\prime}$, the impact law is needed to govern the normal component of the generalized velocity during the collisions, whereas the friction law governs the tangential part indifferently the smooth episodes of motion as well as the collisions. The energetic consistency of this new formulation is proved in full generality (proposition 5.1 at the end of section 5). It is shown in subsection 6.1 that the paradigm of Coulomb friction applying to real world reaction forces offers a heuristic but systematic means to construct the friction sets entering in problem $\mathcal{P}_{4}^{\prime}$. This enables us to analyze in section 6 how this new formulation of the dynamics is compared with the evolution problem based on the paradigm of Coulomb friction applying to reaction forces in the real world in the few cases where such a formulation is available. Such formulations are known to show paradoxes of indeterminacies (Painlevé) and energetic inconsistencies (Kane), and we discuss how these paradoxes are fixed by adopting a formulation along the lines of problem $\mathcal{P}_{4}^{\prime}$.

\section{Background in analytical mechanics and constraints}

Analytical mechanics is a refinement of Newtonian mechanics which was developed by Lagrange in the 18th century. Its basics are briefly recalled in this section with the purpose of fixing the notations for the sequel. 


\subsection{Equation of the dynamics and virtual powers}

The object is that of discrete mechanical systems. A discrete mechanical system is a mechanical system whose arbitrary configuration in the space can be described by a finite number $d$ of independent real numbers: the generalized coordinates denoted by $q^{1}, q^{2}, \ldots, q^{d}$. Here, "independent" means that it is always possible to conceive a motion for which all generalized coordinates but an arbitrary one, remain fixed. The number $d$ is called the number of degrees of freedom of the discrete mechanical system. Discrete mechanical systems generally arise when a finite collection of solids, all of them being modeled as rigid bodies and some of them being possibly connected by so-called perfect joints, is considered. A common application field of discrete mechanical systems is robotics.

The notation $\mathbf{q}=\left(q^{1}, q^{2}, \ldots, q^{d}\right)$ will often be used and $\mathbf{q}$ will be called the abstract configuration (or sometimes simply, the configuration) of the system. A motion of the system is simply a mapping $\mathbf{q}(t)$ defined on some time interval and taking abstract configuration values. As usual, its derivative with respect to time is denoted by $\dot{\mathbf{q}}(t)$ and the vector $\dot{\mathbf{q}}(t)$ is called the generalized velocity at time $t$. The generalized velocity is a convenient mathematical representation of the whole velocity field over the body (or bodies). The kinetic energy $K(\mathbf{q}, \dot{\mathbf{q}})$ is quadratic with respect to the generalized velocity and defines the so-called kinetic matrix $\mathbf{M}(\mathbf{q})$ :

$$
K(\mathbf{q}, \dot{\mathbf{q}})=\frac{1}{2} \dot{\mathbf{q}} \cdot \mathbf{M}(\mathbf{q}) \cdot \dot{\mathbf{q}} .
$$

The kinetic matrix is always symmetric and positive. It is actually positive definite, if the system does not involve any massless components, which will always be assumed in the sequel.

More precisely, the set $Q$ of all abstract configurations is endowed with the structure of a differentiable manifold. This point of view makes it possible to consider an abstract configuration $\mathbf{q}$ with no need of a prior definition of generalized coordinates (that is, a parametrization of the system) and is particularly useful when it is important to distinguish intrinsic quantities, that is quantities not relying on a specific choice of generalized coordinates on the configuration manifold. In this context, the generalized velocities belong to tangent spaces to the configuration manifold. The kinetic matrix endows each tangent space with a scalar product, so that the configuration manifold is actually a Riemannian manifold. When emphasis is to be put on intrinsic quantities, the following alternative notations will be possibly encountered in the sequel:

$$
\left(\dot{\mathbf{q}}_{1}, \dot{\mathbf{q}}_{2}\right)_{\mathbf{q}}=\dot{\mathbf{q}}_{1} \cdot \mathbf{M}(\mathbf{q}) \cdot \dot{\mathbf{q}}_{2}, \quad\|\dot{\mathbf{q}}\|_{\mathbf{q}}=\sqrt{\dot{\mathbf{q}} \cdot \mathbf{M}(\mathbf{q}) \cdot \dot{\mathbf{q}}} .
$$

In any case, the point of view of Riemannian manifold on the configuration space, although enlightening, will not be strictly needed and there will be no harm in identifying in the sequel the configuration manifold with (an open subset of) $\mathbb{R}^{d}$ and the tangent space $T_{\mathbf{q}} Q$ at configuration $\mathbf{q}$, with $\mathbb{R}^{d}$. This identification will be nothing but considering a particular choice of generalized coordinates.

The generalized force $\mathbf{f}$ of Lagrange is defined by means of the virtual power that the internal and external forces develop in any virtual generalized velocity $\hat{\mathbf{v}}$. This virtual power reads as:

$$
\langle\mathbf{f}, \hat{\mathbf{v}}\rangle=f_{i} \hat{v}^{i},
$$

(with the usual convention of summation on repeated indices) and shows that the generalized force belongs to the dual space $T_{\mathbf{q}}^{*} Q$ of the vector space $T_{\mathbf{q}} Q$ of all generalized velocities at configuration $\mathbf{q}$. In particular, the generalized force for a mechanical system with $d$ degrees of freedom has $d$ components. The virtual power point of view is usually used to compute the generalized force from a given distribution of forces $\tilde{\mathbf{f}}(\mathbf{x})$ in the real world (here $\mathbf{x}$ denotes the space variable in the three-dimensional space). First, the real world velocity $\tilde{\mathbf{v}}(\mathbf{x})$ is computed in terms of the generalized velocity $\hat{\mathbf{v}}$ :

$$
\tilde{\mathbf{v}}(\mathbf{x})=\mathbf{l}(\mathbf{q}, \mathbf{x}) \cdot \hat{\mathbf{v}},
$$

where $\mathbf{l}(\mathbf{q}, \mathbf{x}): T_{\mathbf{q}} Q \mapsto \mathbb{R}^{3}$ is a linear mapping depending in general on the current configuration $\mathbf{q}$ and $\mathbf{x}$. Then, the virtual power paradigm:

$$
\forall \hat{\mathbf{v}}, \quad\langle\mathbf{f}, \hat{\mathbf{v}}\rangle=\int \tilde{\mathbf{f}}(\mathbf{x}) \cdot \tilde{\mathbf{v}}(\mathbf{x}) \mathrm{d} \mathbf{x}=\left\langle\int{ }^{\mathrm{t}} \mathbf{l}(\mathbf{q}, \mathbf{x}) \cdot \tilde{\mathbf{f}}(\mathbf{x}) \mathrm{d} \mathbf{x}, \hat{\mathbf{v}}\right\rangle \quad \Longrightarrow \quad \mathbf{f}=\int{ }^{\mathrm{t}} \mathbf{l}(\mathbf{q}, \mathbf{x}) \cdot \tilde{\mathbf{f}}(\mathbf{x}),
$$


provides the expression of the generalized force $\mathbf{f}$ in terms of the real world force distribution $\tilde{\mathbf{f}}(\mathbf{x})$ (here, in the case where $\tilde{\mathbf{f}}(\mathbf{x})$ consists in finitely many point forces, the above integral reduces to a finite sum). Let us point out once more than it is always possible to compute the generalized force from a real world force distribution but that the real world force distribution cannot be recovered in general from the generalized force.

The generalized acceleration $\gamma$ of Lagrange is defined by means of the virtual power it develops in any virtual generalized velocity $\hat{\mathbf{v}}$.

$$
\langle\boldsymbol{\gamma}, \hat{\mathbf{v}}\rangle=\left(\frac{\mathrm{D} \dot{\mathbf{q}}}{\mathrm{D} t}, \hat{\mathbf{v}}\right)_{\mathbf{q}}=\left(\frac{\mathrm{d}}{\mathrm{d} t} \frac{\partial K}{\partial \dot{q}^{i}}-\frac{\partial K}{\partial q^{i}}\right) \hat{v}^{i},
$$

where $\mathrm{D} / \mathrm{D} t$ stands for the covariant derivative along the motion. It satisfies:

$$
\frac{\mathrm{d} K}{\mathrm{~d} t}=\left(\frac{\mathrm{D} \dot{\mathbf{q}}}{\mathrm{D} t}, \dot{\mathbf{q}}\right)_{\mathbf{q}} \text {. }
$$

The fundamental principle of classical dynamics asserts the equation of motion $\gamma=\mathbf{f}$, which is equivalent to the principle of virtual power:

$$
\forall \hat{\mathbf{v}} \in T_{\mathbf{q}} Q, \quad\left(\frac{\mathrm{D} \dot{\mathbf{q}}}{\mathrm{D} t}, \hat{\mathbf{v}}\right)_{\mathbf{q}}=\langle\mathbf{f}, \hat{\mathbf{v}}\rangle .
$$

Remark. The above setting is intrinsic with respect to changes of parametrization, but not with respect to changes of frame of reference. In particular, it has been implicitly assumed that a (galilean) frame of reference has been arbitrarily chosen prior to the formulation of the dynamics. Obtaining a formulation which is also intrinsic with respect to changes of frame of reference requires a higher level of abstraction [8]. Such a framework is not adopted here because the interest is driven to unilateral problems involving impacts. In a formulation that is free of the choice of a frame of reference, it turns out that one can only consider impacts involving bodies of the discrete system under study and one cannot consider the impact of a body of the system onto an external obstacle. Since the latter situation is frequently encountered in practice, we have preferred not to exclude it. Therefore, in all the paper, a particular (galilean) frame of reference is assumed to have been fixed prior to the formulation of the dynamics. For a discussion of the formulation of impact laws that are free of the choice of a frame of reference, the reader is referred to [22].

\subsection{Perfect bilateral constraints}

A bilateral constraint is a restriction on the admissible configurations which can be expressed in terms of a smooth constraint function $\varphi$ as:

$$
\varphi(\mathbf{q})=0 .
$$

The differential of the constraint at $\mathbf{q}$ will be denoted by $\mathrm{d} \varphi_{\mathbf{q}}$. It is a linear form defined on $T_{\mathbf{q}} Q$, that is, an element in the dual space $T_{\mathbf{q}}^{*} Q$. Its components in a given parametrization are the $\partial \varphi / \partial q^{i}$ $(i=1, \ldots, d)$. There may exist several bilateral constraints $\varphi_{\alpha}(\alpha=1,2, \ldots, n)$. In that case, it is usual to make the regularity assumption that the $\mathrm{d} \varphi_{\alpha, \mathbf{q}}$ are linearly independent. This entails, in particular, that there cannot be more than $d$ bilateral constraints: $n \leq d$.

Of course, the realization of some bilateral constraints requires the handling of some (generalized) reaction force $\mathbf{r}$ in the equation of motion: $\gamma=\mathbf{f}+\mathbf{r}$. The constraints are said perfect or ideal if the virtual power of the reaction force $\mathbf{r}$ vanishes in any virtual velocity that is consistent with the realization of the constraints. For perfect bilateral constraints, the reaction force reads as:

$$
\mathbf{r}=\sum_{\alpha=1}^{n} \lambda_{\alpha} \mathrm{d} \varphi_{\alpha, \mathbf{q}}
$$

The regularity assumption on the constraints which requires the linear independence of the $\mathrm{d} \varphi_{\alpha, \mathbf{q}}$ $(\alpha=1,2, \ldots, n)$ implies that the set of all admissible configurations is a manifold of dimension $d-n$. This means that it is possible to take it as the configuration manifold of the system, which is now seen as a system with $d-n$ instead of $d$ degrees of freedom. This point-of-view is that of a so-called reduced 
parametrization and does not involve any reaction force. Of course, the point-of-view of a constrained $d$ d.o.f. system with a reaction force and that of an unconstrained $d-n$ d.o.f. system with reduced parametrization are equivalent in the sense that the predictions of motion are the same.

\subsection{Perfect unilateral constraints and the impact constitutive equation}

Consider a $d$ d.o.f. discrete mechanical system. A unilateral constraint is a restriction on the admissible configurations which can be expressed in terms of a smooth constraint function $\varphi$ as:

$$
\varphi(\mathbf{q}) \geq 0 .
$$

There may exist several unilateral constraints $\varphi_{\alpha}(\alpha=1,2, \ldots, n)$, but they do not need to be simultaneously active (a unilateral constraint $\varphi_{\alpha}$ is said to be active in the configuration $\mathbf{q}$ if that configuration fulfills the equality $\varphi_{\alpha}(\mathbf{q})=0$ in the constraint). The set of all the indices for which the corresponding constraint is active in the configuration $\mathbf{q}$ will be denoted by $J(\mathbf{q})$ :

$$
J(\mathbf{q})=\left\{\alpha \mid \varphi_{\alpha}(\mathbf{q})=0\right\} .
$$

This definition makes it possible to extend the regularity hypothesis made for bilateral constraints to the case of unilateral constraints. The unilateral constraints $\varphi_{\alpha}$ are supposed to be such that, for any admissible configuration $\mathbf{q}$, the $\mathrm{d} \varphi_{\alpha, \mathbf{q}}(\alpha \in J(\mathbf{q}))$ are linearly independent.

As for bilateral constraints, the realization of the constraints involves some reaction force $\mathbf{r}$. The unilateral constraints are said perfect or frictionless, if this reaction force reads as:

$$
\mathbf{r}=\sum_{\alpha=1}^{n} \lambda_{\alpha} \mathrm{d} \varphi_{\alpha, \mathbf{q}},
$$

with $\lambda_{\alpha} \geq 0$ and $\lambda_{\alpha} \varphi_{\alpha}(\mathbf{q}) \equiv 0$. Unlike in the case of bilateral constraints, there remains some indetermination in the equation of motion. Classically, one has to expect that the motion exhibits some velocity jumps that are called impacts or collisions. But, the above equations fail to predict the outcome of an impact. This fact can easily be understood as follows. The outcome of an impact is governed by the deformation waves propagating in the bodies. Since these bodies are modeled as rigid bodies, these waves are not handled by the theory and an indetermination occurs. This indetermination must be fixed by an additional equation: it is called the impact constitutive equation. Its general form is:

$$
\dot{\mathbf{q}}^{+}=\mathcal{F}\left(\mathbf{q}, \dot{\mathbf{q}}^{-}\right)
$$

where $\mathbf{q}$ is the configuration of the system at the impact time, $\dot{\mathbf{q}}^{-}$the left (impacting) velocity and $\dot{\mathbf{q}}^{+}$ the right (outcoming) velocity. The function $\mathcal{F}$ is a datum of the problem. In a practical situation, it has to be identified by using either experiments or a refined theory such as the mechanics of deformable bodies. Usual examples ${ }^{1}$ of such a function $\mathcal{F}$ are the Poisson law or the Moreau law. The impact constitutive equation has exactly the same status as the material constitutive equation in continuum mechanics. It is usual to require that the impact constitutive equation should not increase the kinetic energy during impacts, but energy losses can be allowed (when the impact constitutive equation preserves kinetic energy, it is said to be elastic). The function $\mathcal{F}$ has to fulfill some other restrictions in order to be compatible with the equation of motion. Further restrictions also appear from the frictionless assumption. These restrictions are systematically listed in [2].

\subsection{Well-posedness of the Cauchy problem}

First, the case with no unilateral constraint is recalled. We consider a discrete mechanical with (reduced) configuration manifold $Q$ (supposed to be at least $C^{2}$, as well as the mapping $\mathbf{M}(\mathbf{q})$ and locally identified with $\mathbb{R}^{d}$ ). The generalized force $\mathbf{f}$ is supposed to be given as a function, not only of time, but possibly depending on the current configuration and generalized velocity. Given also the arbitrary initial configuration $\mathbf{q}_{0}$ and initial velocity $\mathbf{v}_{0}$, the Cauchy problem raised by the dynamics reads as follows.

\footnotetext{
${ }^{1} \mathrm{~A}$ critical review of the usual impact laws can be found in [7].
} 
Problem $\mathcal{P}_{1}$. Find $\mathbf{q} \in C^{2}([0, T] ; Q)$ such that:

- $\mathbf{q}(0)=\mathbf{q}_{0}, \quad \dot{\mathbf{q}}(0)=\mathbf{v}_{0}$.

- $\forall t, \quad \mathbf{M}(\mathbf{q}) \cdot \frac{\mathrm{D} \dot{\mathbf{q}}}{\mathrm{D} t}=\mathbf{f}(t ; \mathbf{q}, \dot{\mathbf{q}})$.

The following results about problem $\mathcal{P}_{1}$ are classical.

- If $\mathbf{f}$ is $C^{0}$ (continuous), then there exists a local solution (Peano's theorem) but this local solution does not need to be unique.

- If $\mathbf{f}$ is $C^{1}$, then there exists a local solution. This local solution is unique in the sense that two arbitrary local solutions are identically equal on the time interval where they are both defined. Hence, there exists a unique maximal solution (Cauchy theorem). But, this maximal solution may blow up at a finite time and thus may be not defined all over $[0, T]$.

- If $\mathbf{f}$ is $C^{1}$ and globally Lipschitz-continuous with respect to $(\mathbf{q}, \dot{\mathbf{q}})$ (in terms of the Riemannian distance), then the maximal solution is defined for all times $t$.

Next, the situation where $n$ perfect unilateral constraints are taken into account is considered. The space in which the solution will be sought is the space $M M A([0, T] ; Q)$ (motions with measure acceleration) of those functions whose second distributional derivative is a measure. Every function in $\operatorname{MMA}([0, T] ; Q)$ is a continuous function with left and right derivatives $\dot{\mathbf{q}}^{-}(t), \dot{\mathbf{q}}^{+}(t)$ at each $t$. The functions $\dot{\mathbf{q}}^{-}(t)$ and $\dot{\mathbf{q}}^{+}(t)$ are functions with bounded variation. They are equal for all $t$ except for some $t$ belonging to a countable subset of $[0, T]$. Those $t$ at which $\dot{\mathbf{q}}^{-}(t) \neq \dot{\mathbf{q}}^{+}(t)$ are the instants of impact. The systematic reference on functions of time with bounded variation is [17]. The space of (Radon) measures on $[0, T]$ being denoted by $\mathcal{M}([0, T] ; \mathbb{R})$, the evolution problem associated with the dynamics reads as follows [1].

Problem $\mathcal{P}_{2}$. Find $\mathbf{q} \in M M A([0, T] ; Q)$ and $\lambda_{\alpha} \in \mathcal{M}([0, T] ; \mathbb{R})(\alpha=1, \ldots, n)$ such that:

- $\mathbf{q}(0)=\mathbf{q}_{0}, \quad \dot{\mathbf{q}}(0)=\mathbf{v}_{0} \quad$ (initial condition),

- $\mathbf{M}(\mathbf{q}) \cdot \frac{\mathrm{D} \dot{\mathbf{q}}}{\mathrm{D} t}=\mathbf{f}(t ; \mathbf{q}, \dot{\mathbf{q}})+\sum_{\alpha=1}^{n} \lambda_{\alpha} \mathrm{d} \varphi_{\alpha, \mathbf{q}} \quad$ (equation of motion, in the sense of distributions),

- $\forall \alpha, \quad \varphi_{\alpha}(\mathbf{q}) \geq 0, \quad \lambda_{\alpha} \geq 0, \quad \lambda_{\alpha} \varphi_{\alpha}(\mathbf{q}) \equiv 0 \quad$ (contact conditions),

- $\forall t, \quad \dot{\mathbf{q}}^{+}=\mathcal{F}\left(\mathbf{q}, \dot{\mathbf{q}}^{-}\right) \quad$ (impact constitutive law).

The following comments must be made about the data in problem $\mathcal{P}_{2}$.

- Naturally, the initial configuration $\mathbf{q}_{0}$ has to be compatible with the constraints: $\varphi_{\alpha}\left(\mathbf{q}_{0}\right) \geq 0$, for all $\alpha$. No additional restriction on the initial condition $\left(\mathbf{q}_{0}, \mathbf{v}_{0}\right)$ is prescribed. As a consequence, the velocity $\dot{\mathbf{q}}(0)$ must be understood as a left-velocity: $\dot{\mathbf{q}}(0)=\dot{\mathbf{q}}^{-}(0)$. If the initial condition for the velocity turns out to be incompatible with the unilateral constraint, then an impact will occur at the initial instant and the value of $\dot{\mathbf{q}}^{+}(0) \neq \mathbf{v}_{0}$ will be governed by the impact constitutive law.

- The term $\mathbf{f}(t ; \mathbf{q}, \dot{\mathbf{q}})$ in problem $\mathcal{P}_{2}$ requires a comment since $\dot{\mathbf{q}}$ is not defined at the instants of impact where $\dot{\mathbf{q}}^{-}(t) \neq \dot{\mathbf{q}}^{+}(t)$. However, if the function $\mathbf{f}$ is continuous (which will always be assumed), then the function $t \mapsto \mathbf{f}\left(t ; \mathbf{q}(t), \dot{\mathbf{q}}^{+}(t)\right)$ admits a left and right limit at every instant and these limits are equal except for some $t$ belonging to a countable subset of $[0, T]$. Hence, the function $t \mapsto \mathbf{f}\left(t ; \mathbf{q}(t), \dot{\mathbf{q}}^{+}(t)\right)$ is regulated (is the uniform limit of a sequence of step-functions), is therefore integrable and equals the almost everywhere defined function $\mathbf{f}(t ; \mathbf{q}(t), \dot{\mathbf{q}}(t))$ almost everywhere. Hence, the equation of motion in problem $\mathcal{P}_{2}$ is meaningful.

- The impact constitutive law is supposed to be compatible with the perfect unilateral constraints as in [2]. That is, the function $\mathcal{F}$ must fulfill the following consistency requirements:

$-\forall \mathbf{q}, \dot{\mathbf{q}}^{-}, \quad \forall \alpha \in J(\mathbf{q}), \quad\left\langle\mathrm{d} \varphi_{\alpha, \mathbf{q}}, \mathcal{F}\left(\mathbf{q}, \dot{\mathbf{q}}^{-}\right)\right\rangle_{\mathbf{q}} \geq 0$

(no friction: the post-impact velocity do not violate the constraint), 


$$
-\forall \mathbf{q}, \dot{\mathbf{q}}^{-}, \quad \mathbf{M}(q) \cdot\left(\mathcal{F}\left(\mathbf{q}, \dot{\mathbf{q}}^{-}\right)-\dot{\mathbf{q}}^{-}\right) \in \sum_{\alpha \in J(\mathbf{q})} \mathbb{R}^{+} \mathrm{d} \varphi_{\alpha, \mathbf{q}}
$$

(the generalized reaction force impulse is directed along the normal)

and not to increase the kinetic energy during an impact:

$$
\left\|\mathcal{F}\left(\mathbf{q}, \dot{\mathbf{q}}^{-}\right)\right\|_{\mathbf{q}} \leq\left\|\dot{\mathbf{q}}^{-}\right\|_{\mathbf{q}} .
$$

In this framework, if the mappings $\mathbf{M}(q), \varphi_{\alpha}(\mathbf{q})$ and $\mathbf{f}(t ; \mathbf{q}, \dot{\mathbf{q}})$ are only supposed to be of class $C^{\infty}$, then there may exist multiple solutions for problem $\mathcal{P}_{2}$. However, if these mappings are analytic functions, then there exists a unique maximal solution and, furthermore, if $\mathbf{f}(t ; \mathbf{q}, \dot{\mathbf{q}})$ is globally Lipschitz-continuous with respect to $(\mathbf{q}, \dot{\mathbf{q}})$, then this maximal solution is defined on $[0, T][1,3]$.

Note that the frictionless nature of the unilateral constraints in problem $\mathcal{P}_{2}$ is expressed in terms of the generalized reaction force and that no reference to the forces in the real world is made. This is the price to pay to be able to write a systematic and consistent evolution problem. However, in any situation where a 'naive' formulation in terms of the real world reaction forces is possible, it can be proved that this naive formulation will coincide with problem $\mathcal{P}_{2}$ above.

This consistent formulation of frictionless multibody dynamics in terms of generalized reaction force only, strongly motivates the derivation of a formulation similar to problem $\mathcal{P}_{2}$ for the case of dry friction and will be the object of the following sections.

\section{The case of one point particle with one frictional unilateral constraint}

\subsection{The Coulomb friction law for a point particle}

The Coulomb law of friction is an empirical law based on experiments conducted by Amontons in France at the end of the 17th century and by Coulomb at the beginning of the 19th century. An account of the experiments conducted by Coulomb is to be found in [6].

The principle of the experiments was as follows. Some weights were placed on a plate lying on a horizontal plane table. Then, a horizontal force was applied on the plate and its intensity at onset of sliding was measured. The Coulomb law is an empirical law, designed to provide a reasonable account of these experiments. The intensity of the total weight being denoted by $r_{\mathrm{n}}$, the exerted horizontal force by $\mathbf{r}_{\mathrm{t}}$ and the (horizontal) sliding velocity by $\mathbf{v}_{\mathrm{t}}$, the Coulomb law reads as:

$$
\left\|\mathbf{r}_{\mathrm{t}}\right\| \leq \mu r_{\mathrm{n}} \text { and } \mid \begin{aligned}
& \left\|\mathbf{r}_{\mathrm{t}}\right\|<\mu r_{\mathrm{n}} \Rightarrow \mathbf{v}_{\mathrm{t}}=\mathbf{0}, \\
& \left\|\mathbf{r}_{\mathrm{t}}\right\|=\mu r_{\mathrm{n}} \Rightarrow \mathbf{r}_{\mathrm{t}}=-\lambda \mathbf{v}_{\mathrm{t}} \quad \text { with } \lambda \geq 0,
\end{aligned}
$$

where $\lambda$ is an indeterminate nonnegative real number and where $\mu$ is a dimensionless physical constant measured by Coulomb. It is called the friction coefficient. In a first approximation, it is independent of the total weight $r_{\mathrm{n}}$ and the sliding velocity (in particular, no distinction is made between a dynamical and a static friction coefficient as can be sometimes encountered). However, it depends on the design of the experiment and the materials involved.

In equation (2.1), $\|\cdot\|$ denotes the usual euclidean norm in $\mathbb{R}^{2}$. Classically, the language of convex analysis can be used to rewrite equation (2.1) in a more concise form. Let $\varphi: \mathbb{R}^{2} \rightarrow \mathbb{R} \cup\{+\infty\}$ be a proper (not identically equal to $+\infty$ ), lower semi-continuous, convex function. Its domain $D(\varphi)$ is the (nonempty convex) subset of $\mathbb{R}^{2}$ of those $\mathbf{x}$ where $\varphi$ takes a finite value. The subdifferential $\partial \varphi[\mathbf{x}]$ of $\varphi$ at $\mathbf{x}$ is defined by:

$$
\partial \varphi[\mathbf{x}]=\left\{\mathbf{y} \in \mathbb{R}^{2} \mid \forall \hat{\mathbf{x}} \in \mathbb{R}^{2}, \quad \varphi(\hat{\mathbf{x}}) \geq \varphi(\mathbf{x})+\langle\mathbf{y}, \hat{\mathbf{x}}-\mathbf{x}\rangle\right\}
$$

and the (Legendre-Fenchel) conjugate function $\varphi^{*}$ of $\varphi$ is defined by:

$$
\varphi^{*}(\mathbf{y})=\sup _{\mathbf{x} \in \mathbb{R}^{2}}\{\langle\mathbf{y}, \mathbf{x}\rangle-\varphi(\mathbf{x})\} .
$$


It is a proper, lower semi-continuous, convex function. The fundamental result about the LegendreFenchel transform is:

$$
\mathbf{y} \in \partial \varphi[\mathbf{x}] \quad \Longleftrightarrow \quad \mathbf{x} \in \partial \varphi^{*}[\mathbf{y}] \quad \Longleftrightarrow\langle\mathbf{x}, \mathbf{y}\rangle=\varphi(\mathbf{x})+\varphi^{*}(\mathbf{y})
$$

Let $\mathcal{C}$ be a nonempty closed convex subset of $\mathbb{R}^{2}$. Its indicatrix function $I_{\mathcal{C}}$ is defined by:

$$
I_{\mathcal{C}}(\mathbf{x})=\mid \begin{array}{ll}
0 & \text { if } \mathbf{x} \in \mathcal{C} \\
+\infty & \text { if } \mathbf{x} \notin \mathcal{C}
\end{array}
$$

It is proper, lower semi-continuous and convex. Its conjugate function (by the Legendre-Fenchel transform) is the support function $S_{\mathcal{C}}$ of $\mathcal{C}$ :

$$
S_{\mathcal{C}}(\mathbf{y})=\sup _{\mathbf{x} \in \mathcal{C}}\langle\mathbf{x}, \mathbf{y}\rangle .
$$

Introducing the closed unit disk $\mathcal{B}$ of $\mathbb{R}^{2}$, it is readily checked that:

$$
S_{\mathcal{B}}(\mathbf{y})=\|\mathbf{y}\| \text {. }
$$

The Coulomb law (2.1) is therefore equivalent to anyone of the following equivalent statements:

$$
\begin{array}{ll}
\text { (i) } & -\mathbf{v}_{\mathrm{t}} \in \partial I_{\mu r_{\mathrm{n}} \mathcal{B}}\left[\mathbf{r}_{\mathrm{t}}\right] \\
\text { (ii) } \mathbf{r}_{\mathrm{t}} \in \partial S_{\mu r_{\mathrm{n}} \mathcal{B}}\left[-\mathbf{v}_{\mathrm{t}}\right] \\
\text { (iii) } \forall \hat{\mathbf{v}} \in \mathbb{R}^{2}, \quad\left\langle\mathbf{r}_{\mathrm{t}}, \hat{\mathbf{v}}-\mathbf{v}_{\mathrm{t}}\right\rangle+\mu r_{\mathrm{n}}\left(\|\hat{\mathbf{v}}\|-\left\|\mathbf{v}_{\mathrm{t}}\right\|\right) \geq 0 .
\end{array}
$$

Since $\partial I_{\mathcal{C}}$ is the closed convex cone of all the outward normal vectors, statement (i) is also called the normality rule, when $\mu r_{\mathrm{n}}$ is assumed to be given as in Coulomb's experiments. The equivalent statement (ii), is often referred to as the maximum dissipation principle.

\subsection{The dynamics of a point particle with one frictional unilateral constraint}

We consider a point particle of unit mass whose instantaneous location is given by $\mathbf{q}(t) \in \mathbb{R}^{d}$ ( $d$ equals 2 or 3$)$. A force $\mathbf{f}(t ; \mathbf{q}, \dot{\mathbf{q}})$ is supposed to be exerted on the particle. This particle is assumed to obey the unilateral constraint $\varphi(\mathbf{q}) \geq 0$ where $\varphi: \mathbb{R}^{d} \mapsto \mathbb{R}$ is a smooth function whose differential is supposed to vanish nowhere ${ }^{2}: \mathrm{d} \varphi_{\mathbf{q}} \neq \mathbf{0}$.

Any vector $\mathbf{v}$ based at location $\mathbf{q}$ admits a decomposition into normal and tangential parts with respect to the function $\varphi$ :

$$
\mathbf{v}=v_{\mathrm{n}} \mathbf{n}+\mathbf{v}_{\mathrm{t}}, \quad \text { with: } \quad \mathbf{n}=\nabla \varphi_{\mathbf{q}} /\left\|\nabla \varphi_{\mathbf{q}}\right\|, \quad v_{\mathrm{n}}=\langle\mathbf{v}, \mathbf{n}\rangle, \quad \mathbf{v}_{\mathrm{t}}=\mathbf{v}-v_{\mathrm{n}} \mathbf{n},
$$

where $\|\cdot\|$ and $\langle\cdot, \cdot\rangle$ stand for the usual euclidean norm and scalar product in $\mathbb{R}^{d}$. The tangent hyperspace to the constraint at $\mathbf{q}$ is denoted by $\mathcal{T}_{\mathbf{q}}$ :

$$
\mathcal{T}_{\mathbf{q}}=\left\{\mathbf{v} \in \mathbb{R}^{d} \mid\left\langle\mathrm{d} \varphi_{\mathbf{q}}, \mathbf{v}\right\rangle=0\right\}
$$

The evolution problem associated with the dynamics of the particle reads as follows.

Problem $\mathcal{P}_{3}$. Find $\mathbf{q} \in M M A\left([0, T] ; \mathbb{R}^{d}\right)$ and $\mathbf{r} \in \mathcal{M}\left([0, T] ; \mathbb{R}^{d}\right)$ such that:

- $\mathbf{q}(0)=\mathbf{q}_{0}, \quad \dot{\mathbf{q}}(0)=\mathbf{v}_{0} \quad$ (initial condition),

- $\ddot{\mathbf{q}}=\mathbf{f}(t ; \mathbf{q}, \dot{\mathbf{q}})+\mathbf{r} \quad$ (equation of motion, in the sense of distributions),

- $\varphi(\mathbf{q}) \geq 0, \quad r_{\mathrm{n}} \geq 0, \quad r_{\mathrm{n}} \varphi(\mathbf{q}) \equiv 0 \quad$ (contact conditions),

- $\forall \hat{\mathbf{v}} \in C^{0}\left([0, T] ; \mathcal{T}_{\mathbf{q}}\right), \quad \int_{[0, T]}\left\langle\mathbf{r}_{\mathrm{t}}, \hat{\mathbf{v}}-\dot{\mathbf{q}}_{\mathrm{t}}^{+}\right\rangle+\mu r_{\mathrm{n}}\left(\|\hat{\mathbf{v}}\|-\left\|\dot{\mathbf{q}}_{\mathrm{t}}^{+}\right\|\right) \geq 0$

(Coulomb friction),

- $\varphi(\mathbf{q}(t))=0 \quad \Longrightarrow \quad \dot{q}_{\mathrm{n}}^{+}(t)=-e\left(\mathbf{q}, \dot{\mathbf{q}}^{-}\right) \dot{q}_{\mathrm{n}}^{-}(t)$

(impact constitutive equation).

${ }^{2}$ Actually, it only needs to vanish nowhere in a neighborhood of the hypersurface of equation $\varphi(\mathbf{x})=0$. 
In problem $\mathcal{P}_{3}, \mu \geq 0$ is the given friction coefficient. The weak form adopted for the Coulomb friction law is suitable for the case where the reaction force $\mathbf{r}$ is a measure and makes sense for the smooth episodes of motion as well as for the impacts (recall that the function $\dot{\mathbf{q}}_{\mathbf{t}}^{+}$has bounded variation and is therefore integrable with respect to any measure such as $\mathbf{r}_{\mathrm{t}}$ and the same is true of the continuous function $\hat{\mathbf{v}}$ ). The modulus measure of the measure $\mathbf{r}_{\mathrm{t}}$ being denoted by $\left\|\mathbf{r}_{\mathrm{t}}\right\|$, the friction law entails that $\left\|\mathbf{r}_{\mathrm{t}}\right\| \leq \mu r_{\mathrm{n}}$ in the sense of the real measure ordering. One consequence is that a jump in the tangential velocity $\left(\mathbf{q}_{\mathrm{t}}^{+}(t) \neq \mathbf{q}_{\mathrm{t}}^{-}(t)\right)$ entails a jump in the normal velocity $\left(q_{\mathrm{n}}^{+}(t) \neq q_{\mathrm{n}}^{-}(t)\right)$. In the impact constitutive equation, the given restitution coefficient $e\left(\mathbf{q}, \dot{\mathbf{q}}^{-}\right)$is supposed to take values in $[0,1]$. Note that the friction law is written in terms of the right-velocity. As a consequence, it is an easy exercise to check that the impact constitutive equation complemented with the Coulomb friction law completely determines the outcome of an impact (that is, fully determines the right velocity $\dot{\mathbf{q}}^{+}$). It is also readily checked that the kinetic energy always decreases during an impact, as should be expected.

Problem $\mathcal{P}_{3}$ was already considered (in the case of the completely inelastic impact law $e=0$ ) by Monteiro-Marques in [14] where a proof of existence of solution can be found. It is proved in addition in [5] that, if $\varphi$ and $f$ are analytic functions and $\mathbf{f}(t ; \mathbf{q}, \dot{\mathbf{q}})$ is globally Lipschitz-continuous with respect to $(\mathbf{q}, \dot{\mathbf{q}})$, then problem $\mathcal{P}_{3}$ has a unique solution.

\section{The case of a discrete mechanical system with one frictional unilateral constraint}

In this section, we consider a discrete mechanical system with (abstract) configuration denoted by $\mathbf{q}=\left(q^{1}, q^{2}, \ldots, q^{d}\right)$. It is supposed to obey the unilateral constraint $\varphi(\mathbf{q}) \geq 0$. To formulate the evolution problem associated with the situation where dry friction must be associated with that unilateral constraint, it is now proposed to mimic the case of the point particle as in section 2.2.

\subsection{Definition of normal and tangential reaction}

The first step is to obtain a decomposition of generalized velocities and generalized forces into normal and tangential parts. There is only one scalar product for generalized velocities which is intrinsic, that is, which does not rely on the choice of a specific parametrization: the scalar product induced by the kinetic energy. Since the scalar product is associated with the kinetic matrix $\mathbf{M}(\mathbf{q})$, one must now carefully distinguish between the differential $\mathrm{d} \varphi$ and the gradient $\nabla \varphi$ :

$$
\forall \hat{\mathbf{v}}, \quad\left\langle\mathrm{d} \varphi_{\mathbf{q}}, \hat{\mathbf{v}}\right\rangle=\left(\nabla \varphi_{\mathbf{q}}, \hat{\mathbf{v}}\right)_{\mathbf{q}}, \quad \Longrightarrow \quad \nabla \varphi_{\mathbf{q}}=\mathbf{M}^{-1}(\mathbf{q}) \cdot \mathrm{d} \varphi_{\mathbf{q}}
$$

With this definition of the gradient, the decomposition of the generalized velocity $\mathbf{v}$ into tangential and normal parts with respect to the function $\varphi$, reads as:

$$
\begin{aligned}
\mathbf{n} & =\nabla \varphi_{\mathbf{q}} /\left\|\nabla \varphi_{\mathbf{q}}\right\|_{\mathbf{q}}, \\
\mathbf{v}=v_{\mathrm{n}} \mathbf{n}+\mathbf{v}_{\mathrm{t}}, \text { with: } \quad v_{\mathrm{n}} & =(\mathbf{v}, \mathbf{n})_{\mathbf{q}}=\frac{\left\langle\mathrm{d} \varphi_{\mathbf{q}}, \mathbf{v}\right\rangle}{\left\|\mathrm{d} \varphi_{\mathbf{q}}\right\|_{\mathbf{q}}^{*}}=\frac{\left\langle\mathrm{d} \varphi_{\mathbf{q}}, \mathbf{v}\right\rangle}{\sqrt{\mathrm{d} \varphi_{\mathbf{q}} \cdot \mathbf{M}^{-1}(\mathbf{q}) \cdot \mathrm{d} \varphi_{\mathbf{q}}}},
\end{aligned}
$$

where we recall:

$$
\left\langle\mathrm{d} \varphi_{\mathbf{q}}, \mathbf{v}\right\rangle=\frac{\partial \varphi}{\partial q^{i}} v^{i}
$$

This definition gets its consistency from the fact that the normal component $v_{\mathrm{n}}$ of the velocity $\mathbf{v}=\dot{\mathbf{q}}$ vanishes identically during a motion episode where the constraint $\varphi$ remains active:

$$
\forall t, \varphi(\mathbf{q}(t))=0 \quad \Leftrightarrow \quad\left\langle\mathrm{d} \varphi_{\mathbf{q}}, \mathbf{v}\right\rangle \equiv 0 \quad \Leftrightarrow \quad v_{\mathrm{n}} \equiv 0 .
$$

Since the generalized force of Lagrange is defined by means of a virtual power, generalized forces belong to the dual space to the space of generalized velocities. In the space of generalized forces, there is only one intrinsic scalar product: the dual scalar product. The dual scalar product is associated with 
the inverse $\mathbf{M}^{-1}(\mathbf{q})$ of the kinetic matrix. Therefore, the decomposition into a tangential and a normal part for the generalized force $\mathbf{f}$ with respect to the function $\varphi$, reads as:

$$
\begin{aligned}
& \mathbf{f}=f_{\mathrm{n}} \mathbf{n}^{*}+\mathbf{f}_{\mathrm{t}}, \text { with: } \begin{array}{l}
\mathrm{d} \varphi_{\mathbf{q}} /\left\|\mathrm{d} \varphi_{\mathbf{q}}\right\|_{\mathbf{q}}^{*}, \\
f_{\mathrm{n}}
\end{array}=\left(\mathbf{f}, \mathbf{n}^{*}\right)_{\mathbf{q}}^{*}=\frac{\left(\mathrm{d} \varphi_{\mathbf{q}}, \mathbf{f}\right)_{\mathbf{q}}^{*}}{\left\|\mathrm{~d} \varphi_{\mathbf{q}}\right\|_{\mathbf{q}}^{*}}=\frac{\mathrm{d} \varphi_{\mathbf{q}} \cdot \mathbf{M}^{-1}(\mathbf{q}) \cdot \mathbf{f}}{\sqrt{\mathrm{d} \varphi_{\mathbf{q}} \cdot \mathbf{M}^{-1}(\mathbf{q}) \cdot \mathrm{d} \varphi_{\mathbf{q}}}} .
\end{aligned}
$$

This definition gets its consistency from the fact that a generalized force $\mathbf{f}$ has a vanishing tangential component if and only if its virtual power in any tangential virtual velocity vanishes:

$$
\begin{aligned}
\mathbf{f}_{\mathrm{t}}=0 & \Leftrightarrow \forall \hat{\mathbf{v}} \text { such that }\left\langle\mathrm{d} \varphi_{\mathbf{q}}, \hat{\mathbf{v}}\right\rangle=0, \quad\langle\mathbf{f}, \hat{\mathbf{v}}\rangle=0, \\
& \Leftrightarrow \exists \lambda \in \mathbb{R}, \quad \mathbf{f}=\lambda \mathrm{d} \varphi_{\mathbf{q}} .
\end{aligned}
$$

The hyperspace of all tangential generalized velocities at the current configuration $\mathbf{q}$ will be denoted by $\mathcal{T}_{\mathbf{q}} \subset T_{\mathbf{q}} Q$ and the hyperspace of all tangential generalized forces at $\mathbf{q}$ by $\mathcal{T}_{\mathbf{q}}^{*} \subset T_{\mathbf{q}}^{*} Q$. The above equivalence shows that $\mathcal{T}_{\mathbf{q}}^{*}$ can be identified with the dual space of $\mathcal{T}_{\mathbf{q}}$, showing the consistency of the notations.

\subsection{Formulation of the friction law in terms of the generalized reaction}

Making use of the above definitions, the Coulomb friction law (2.1) for the point particle can now be naturally extended to the case of an arbitrary discrete mechanical system with one unilateral constraint $\varphi$. The equation of motion is complemented with a generalized reaction force $\mathbf{r}: \gamma=\mathbf{f}+\mathbf{r}$ and the reaction force $\mathbf{r}$ is split into normal and tangential components: $\mathbf{r}=r_{\mathrm{n}} \mathbf{n}^{*}+\mathbf{r}_{\mathrm{t}}$ with respect to the constraint $\varphi$, according to section 3.1.

The normal reaction $r_{\mathrm{n}}$ is required to be nonnegative and vanishes when the constraint is not active (that is, when $\varphi(\mathbf{q})>0$ ). To generalize the Coulomb friction law (2.1) for the point particle to the case of an arbitrary discrete mechanical system, we are naturally led to postulate the existence of a set $\mathcal{C}_{\mathbf{q}}^{*}$ such that all the admissible tangential components of the reaction force are characterized by the condition:

$$
\mathbf{r}_{\mathrm{t}} \in r_{\mathrm{n}} \mathcal{C}_{\mathbf{q}}^{*}
$$

The set $\mathcal{C}_{\mathbf{q}}^{*}$ is therefore a given subset of the space $\mathcal{T}_{\mathbf{q}}^{*} \subset T_{\mathbf{q}}^{*} Q$ of all tangential generalized forces at the current configuration q. As for the Coulomb friction law (2.1), this condition entails that whenever the normal reaction force vanishes (for example in motion episodes where the constraint is not active), then the tangential reaction also vanishes.

Actually, the Coulomb friction law (2.1) for the point particle is recovered by choosing a disk for the set $\mathcal{C}_{\mathbf{q}}^{*}: \mathcal{C}_{\mathbf{q}}^{*}=\mu \mathcal{B}$. Note that Coulomb conducted his experiments not on point particles, but on plates of finite dimensions. The law was actually formulated using the normal and tangential components of the total force and not using the whole distribution of surface forces (which are indeterminate in the case where the plate is modelled as a rigid body). This total force is nothing but a generalized force and the above point of view is nothing but a natural generalization of Coulomb's original analysis of his own experiments. The systematic construction of the friction set $\mathcal{C}_{\mathbf{q}}^{*}$ in practical examples will be discussed in section 6 .

In general, the set $\mathcal{C}_{\mathbf{q}}^{*}$ will not be required to be a disk (case of isotropic friction), but only to be nonempty. It is also required to be closed and convex so that the normality rule can be adopted as a flow rule: this is nothing but assuming that for a given value of the reaction normal component, the flow rule should obey the maximum dissipation principle ${ }^{3}$. With this assumption, the flow rule is formally expressed by anyone of the following equivalent statements:

$$
\begin{aligned}
& \text { (i) }-\dot{\mathbf{q}}_{\mathrm{t}}^{+} \in \partial I_{r_{\mathrm{n}} \mathcal{C}_{\mathbf{q}}^{*}}\left[\mathbf{r}_{\mathrm{t}}\right] \\
& \text { (ii) } \mathbf{r}_{\mathrm{t}} \in \partial S_{r_{\mathrm{n}} \mathcal{C}_{\mathbf{q}}^{*}}\left[-\dot{\mathbf{q}}_{\mathrm{t}}^{+}\right] \\
& \text {(iii) } \forall \hat{\mathbf{v}} \in \mathcal{T}_{\mathbf{q}}, \quad\left\langle\mathbf{r}_{\mathrm{t}}, \hat{\mathbf{v}}-\dot{\mathbf{q}}_{\mathrm{t}}^{+}\right\rangle+r_{\mathrm{n}}\left[S_{\mathcal{C}_{\mathbf{q}}^{*}}(-\hat{\mathbf{v}})-S_{\mathcal{C}_{\mathbf{q}}^{*}}\left(-\dot{\mathbf{q}}_{\mathrm{t}}^{+}\right)\right] \geq 0,
\end{aligned}
$$

\footnotetext{
${ }^{3}$ it should be said that the adoption of the normality rule in this context is mainly a matter of convenience. A critical discussion of this hypothesis can be found in [13].
} 
where the identity $S_{r_{\mathrm{n}} \mathcal{C}_{\mathbf{q}}^{*}}=r_{\mathrm{n}} S_{\mathcal{C}_{\mathbf{q}}^{*}}$ has been used. Here, the subdifferentials should be understood in the sense of the duality between the space $\mathcal{T}_{\mathbf{q}}$ of tangential generalized velocities and the space $\mathcal{T}_{\mathbf{q}}^{*}$ of tangential generalized forces.

Actually, the set $\mathcal{C}_{\mathbf{q}}^{*}$ can be viewed either as a subset of the space $\mathcal{T}_{\mathbf{q}}^{*}$ of tangential generalized forces or as a subset of the space $T_{\mathbf{q}}^{*} Q$ of all generalized forces. In the latter case, one has:

$$
\forall \hat{\mathbf{v}} \in T_{\mathbf{q}} Q, \quad S_{\mathcal{C}_{\mathbf{q}}^{*}}(\hat{\mathbf{v}})=S_{\mathcal{C}_{\mathbf{q}}^{*}}\left(\hat{\mathbf{v}}_{\mathbf{t}}\right),
$$

since $\mathcal{C}_{\mathbf{q}}^{*}$ is contained in the space $\mathcal{T}_{\mathbf{q}}^{*}$ of tangential generalized forces. Therefore, statement (iii) is equivalent to:

$$
\left(i i i^{\prime}\right) \quad \forall \hat{\mathbf{v}} \in T_{\mathbf{q}} Q, \quad\left\langle\mathbf{r}_{\mathrm{t}}, \hat{\mathbf{v}}-\dot{\mathbf{q}}^{+}\right\rangle+r_{\mathrm{n}}\left[S_{\mathcal{C}_{\mathbf{q}}^{*}}(-\hat{\mathbf{v}})-S_{\mathcal{C}_{\mathbf{q}}^{*}}\left(-\dot{\mathbf{q}}^{+}\right)\right] \geq 0,
$$

where we recall that $T_{\mathbf{q}} Q$ stands for the space of all generalized velocities at the generalized configuration $\mathbf{q}$ (tangent space at $\mathbf{q}$ to the configuration manifold). This latter form of the (generalized) friction law is the more appropriate one in view of deriving an expression of the friction law encompassing the episodes of smooth motion and the impacts, that is, in situations where the reaction force is not pointwise defined but is only a measure (with respect to time). Having these situations in mind, the friction law has to be postulated under the form:

$$
\forall \hat{\mathbf{v}} \in C^{0}\left([0, T] ; T_{\mathbf{q}} Q\right), \quad \int_{[0, T]}\left\langle\mathbf{r}_{\mathrm{t}}, \hat{\mathbf{v}}-\dot{\mathbf{q}}^{+}\right\rangle+r_{\mathrm{n}}\left[S_{\mathcal{C}_{\mathbf{q}}^{*}}(-\hat{\mathbf{v}})-S_{\mathcal{C}_{\mathbf{q}}^{*}}\left(-\dot{\mathbf{q}}^{+}\right)\right] \geq 0 .
$$

We have to check that the generalized friction law (3.1) makes sense in cases where $\mathbf{r}$ is an arbitrary vector-valued measure (with respect to time) and $\dot{\mathbf{q}}^{+}$is an arbitrary (vector-valued) function with bounded variation. This discussion is going to be performed in the restrictive setting in which there is a parametrization of the system for which $\mathcal{C}_{\mathbf{q}}^{*}$ does not depend on $\left(q^{1}, q^{2}, \ldots, q^{d}\right)$, as for the Coulomb law where $\mathcal{C}_{\mathbf{q}}^{*}$ is a disk of radius $\mu$. In more general situations, some assumptions must be stated regarding the regularity of the mapping $\mathbf{q} \mapsto \mathcal{C}_{\mathbf{q}}^{*}$. In a first step, we shall first consider the case where $\mathcal{C}_{\mathbf{q}}^{*}$ is bounded. However, it will be seen in section 6 that it may be desirable to admit unbounded sets $\mathcal{C}_{\mathbf{q}}^{*}$ in some situations, so that we shall discuss in a second step, the case where $\mathcal{C}_{\mathbf{q}}^{*}$ is possibly unbounded.

1. In case where $\mathcal{C}_{\mathbf{q}}^{*}$ is bounded, $S_{\mathcal{C}_{\mathbf{q}}^{*}}$ is finite everywhere and continuous. Let us consider arbitrary $\mathbf{x}$ and $\mathbf{y}$ such that: $\mathbf{y} \in \partial S_{\mathcal{C}_{\mathbf{q}}^{*}}[\mathbf{x}]$. This is equivalent to $\mathbf{x} \in \partial I_{\mathcal{C}_{\mathbf{q}}^{*}}[\mathbf{y}]$ which entails that $\mathbf{y} \in \mathcal{C}_{\mathbf{q}}^{*}$. By hypothesis, there exists $M \in \mathbb{R}^{+}$, independent of $\mathbf{y}$, so that $\|\mathbf{y}\| \leq M$. By an appropriate generalization of the mean-value theorem, this implies that $S_{\mathcal{C}_{\mathbf{q}}^{*}}$ is Lipschitz-continuous of modulus $M$. Therefore, the function $t \mapsto S_{\mathcal{C}_{\mathbf{q}}^{*}}\left(-\dot{\mathbf{q}}^{+}\right)$has bounded variation. We recall that a function with bounded variation, being the uniform limit of a sequence of step-functions, is integrable with respect to any (Radon) measure. The integral in the generalized friction law (3.1) is therefore defined for any (vector-valued) measure $\mathbf{r}$ and any function $\dot{\mathbf{q}}^{+}$with bounded variation.

2. Consider the case where $\mathcal{C}_{\mathbf{q}}^{*}$ is unbounded. In that case, $S_{\mathcal{C}_{\mathbf{q}}^{*}}$ takes the value $+\infty$ and it must be checked that the generalized friction law (3.1) still makes sense. We shall consider only the case where $\mathbf{0} \in \mathcal{C}_{\mathbf{q}}^{*}$ for the sake of simplicity, so that $S_{\mathcal{C}_{\mathbf{q}}^{*}} \geq 0$, but this hypothesis is nothing essential and could be easily removed. The contact condition requires that the measure $r_{\mathrm{n}}$ be nonnegative. It can be proved that the function $t \mapsto S_{\mathcal{C}_{\mathbf{q}}^{*}}(-\hat{\mathbf{v}})$ is a measurable function with values in $[0,+\infty]$. Therefore, the integral:

$$
\int_{[0, T]} r_{\mathrm{n}} S_{\mathcal{C}_{\mathbf{q}}^{*}}(-\hat{\mathbf{v}}) \in[0,+\infty],
$$

makes sense as either a nonnegative real number or $+\infty$, according to the theory of integrals of measurable nonnegative functions with respect to a nonnegative Radon measure. According to that theory, the value of the integral is the same if the integration is performed over $[0, T]$ or over supp $r_{\mathrm{n}}$, which means roughly that the instants where $r_{\mathrm{n}}$ vanishes do not contribute to the value of the integral (although the function $S_{\mathcal{C}_{\mathbf{q}}^{*}}(-\hat{\mathbf{v}})$ can equal $+\infty$ at these instants). This fact 
is consistent with the fact that $r_{\mathrm{n}} S_{\mathcal{C}_{\mathbf{q}}^{*}}$ arises from $S_{r_{\mathrm{n}} \mathcal{C}_{\mathbf{q}}^{*}}$ and that $S_{0 \mathcal{C}_{\mathbf{q}}^{*}}=S_{\{0\}} \equiv 0$. Similarly, the integral

$$
\int_{[0, T]} r_{\mathrm{n}} S_{\mathcal{C}_{\mathbf{q}}^{*}}\left(-\dot{\mathbf{q}}^{+}\right)
$$

is well-defined as an element of $[0,+\infty]$. But, taking the zero constant function as a trial function $\hat{\mathbf{v}}$ in the generalized friction law (3.1) shows that this integral must be finite. Since it is finite, the whole integral in formula (3.1) is well-defined for all trial functions $\hat{\mathbf{v}} \in C^{0}\left([0, T] ; T_{\mathbf{q}} Q\right)$. Actually, the finiteness of the integral (3.2) is a consequence of the generalized friction law and expresses that some values of the sliding velocity are excluded when contact is firmly achieved:

$$
\text { for } r_{\mathrm{n}} \text {-a.a. } t \in \operatorname{supp} r_{\mathrm{n}}, \quad-\mathbf{v}^{+} \in \overline{D\left(S_{\mathcal{C}_{\mathbf{q}}^{*}}\right)}
$$

In other terms, unbounded sets $\mathcal{C}_{\mathbf{q}}^{*}$ forbid some values for the sliding right-velocity during firm contact episodes.

To summarize, the generalized friction law (3.1) makes sense with $\mathbf{r}$ being an arbitrary Radon measure, $\dot{\mathbf{q}}^{+}$an arbitrary function with bounded variation and for $\mathcal{C}_{\mathbf{q}}^{*}$ an arbitrary nonempty closed convex subset of $\mathcal{T}_{\mathbf{q}}^{*}$, either bounded or unbounded.

The fact that unbounded $\mathcal{C}_{\mathbf{q}}^{*}$ are admitted, has some interesting consequences. To get deeper insight into this situation, let us examine the case of a point particle moving on one side of a straight obstacle (straight means that one can choose a linear form as the function $\varphi$ describing its geometry). Its dynamical evolution is governed by problem $\mathcal{P}_{3}$. But in order to analyse the consequences of having unbounded sets $\mathcal{C}_{\mathbf{q}}^{*}$, the Coulomb friction law in problem $\mathcal{P}_{3}$ is replaced by the generalized friction law (3.1) where the set $\mathcal{C}_{\mathbf{q}}^{*}$ is supposed to be unbounded. As already mentioned above, this entails $\overline{D\left(S_{\mathcal{C}_{\mathbf{q}}^{*}}\right)} \neq T_{\mathbf{q}} Q$ and velocities in $T_{\mathbf{q}} Q \backslash \overline{D\left(S_{\mathcal{C}_{\mathbf{q}}^{*}}\right)}$ are excluded by the generalized friction law during firm contact episodes. Now, let us consider a motion of the particle which is free of external forces and achieves grazing contact $\left(r_{\mathrm{n}}=0\right)$ with the obstacle at constant velocity $\mathbf{v}_{0} \in T_{\mathbf{q}} Q \backslash \overline{D\left(S_{\mathcal{C}_{\mathbf{q}}^{*}}\right)}$. Such a motion is associated with $\mathbf{r}=\mathbf{0}$ and satisfies the generalized friction law. Suppose that the normal component of the external force, which is assumed to vanish on $[0, T]$, starts to decrease at $t=T$, so that it is negative for $t>T$. By the impact law $\dot{q}_{\mathrm{n}}^{+}(T)=0$ and the particle must remain in contact with the obstacle for $t>T$. Therefore, the normal component $r_{\mathrm{n}}$ of the reaction force equals the opposite of the normal external force and the contact is firm. But, since the velocity on $[0, T]$ has a value which is excluded by the friction law when contact is firmly achieved, a jump in the tangential velocity must occur at $t=T$. It can be checked that the friction law completely determines the value of the outcoming velocity $\dot{\mathbf{q}}_{t}^{+}(T)$. Therefore, unbounded sets $\mathcal{C}_{\mathbf{q}}^{*}$ allow jumps in tangential velocity with continuous normal velocity. This phenomenon was proved in section 2.2 to be excluded in the case of a bounded $\mathcal{C}_{\mathbf{q}}^{*}$, and is therefore specific to the case of an unbounded $\mathcal{C}_{\mathbf{q}}^{*}$. It is called a tangential impact and will be discussed more thoroughly in section 6.2 where the reason why it is desirable to admit unbounded sets $\mathcal{C}_{\mathbf{q}}^{*}$ as well as tangential impacts will be explained. Tangential impacts have the slightly unpleasant consequence that the value of $\dot{\mathbf{q}}$ in $f_{\mathrm{n}}$ (normal component of $\mathbf{f}$ ) influences the outcome of the tangential impact. Therefore, it matters to choose $\mathbf{f}\left(t ; \mathbf{q}, \dot{\mathbf{q}}^{-}\right)$or $\mathbf{f}\left(t ; \mathbf{q}, \dot{\mathbf{q}}^{+}\right)$in the equation of motion (this choice has consequences only on the tangential impacts). We therefore make arbitrarily the choice of the former to account for possibly unbounded sets $\mathcal{C}_{\mathbf{q}}^{*}$ (the latter choice generating possible indeterminacy in tangential impacts).

The final form (3.1) of the generalized friction law makes it possible to obtain the generalization of the evolution problem $\mathcal{P}_{3}$ that governs the dynamics of a point particle submitted to one frictional unilateral constraint (for which existence and uniqueness of a solution was studied in [5]), to the case of an arbitrary discrete mechanical system subjected to one frictional unilateral constraint $\varphi$. This problem reads formally as follows.

Problem $\mathcal{P}_{3}^{\prime}$. Find $\mathbf{q} \in M M A([0, T] ; Q)$ and $\mathbf{r} \in \mathcal{M}\left([0, T] ; T^{*} Q\right)$ such that:

- $\mathbf{q}(0)=\mathbf{q}_{0}, \quad \dot{\mathbf{q}}(0)=\mathbf{v}_{0} \quad$ (initial condition), 
- $\mathbf{M}(\mathbf{q}) \cdot \frac{\mathrm{D} \dot{\mathbf{q}}}{\mathrm{D} t}=\mathbf{f}\left(t ; \mathbf{q}, \dot{\mathbf{q}}^{-}\right)+\mathbf{r} \quad$ (equation of motion, in the sense of distributions),

- $\mathbf{r}=r_{\mathrm{n}} \mathbf{n}^{*}+\mathbf{r}_{\mathrm{t}}$, with: $\left(\mathbf{r}_{\mathrm{t}}, \mathbf{n}^{*}\right)_{\mathbf{q}}^{*}=0$

(split of the reaction force into normal and tangential components),

- $\varphi(\mathbf{q}) \geq 0, \quad r_{\mathrm{n}} \geq 0, \quad r_{\mathrm{n}} \varphi(\mathbf{q}) \equiv 0 \quad$ (contact conditions),

- $\forall \hat{\mathbf{v}} \in C^{0}\left([0, T] ; T_{\mathbf{q}} Q\right), \quad \int_{[0, T]}\left\langle\mathbf{r}_{\mathrm{t}}, \hat{\mathbf{v}}-\dot{\mathbf{q}}^{+}\right\rangle+r_{\mathrm{n}}\left[S_{\mathcal{C}_{\mathbf{q}}^{*}}(-\hat{\mathbf{v}})-S_{\mathcal{C}_{\mathbf{q}}^{*}}\left(-\dot{\mathbf{q}}^{+}\right)\right] \geq 0$

(generalized friction law),

- $\varphi(\mathbf{q}(t))=0 \quad \Longrightarrow \quad \dot{q}_{\mathrm{n}}^{+}(t)=-e\left(\mathbf{q}, \dot{\mathbf{q}}^{-}\right) \dot{q}_{\mathrm{n}}^{-}(t) \quad$ (impact constitutive equation).

The only difference with the case of the point particle (problem $\mathcal{P}_{3}$ ) is that a friction set $\mathcal{C}_{\mathbf{q}}^{*}$ must be given. Problem $\mathcal{P}_{3}$ is recovered from problem $\mathcal{P}_{3}^{\prime}$ by taking $\mathcal{C}_{\mathbf{q}}^{*}$ as the closed disk of radius $\mu$. The frictionless situation, as formulated in [15] or [1] is recovered by taking $\mathcal{C}_{\mathbf{q}}^{*}=\{\mathbf{0}\}$.

Let us point out that problem $\mathcal{P}_{3}^{\prime}$ is only the general form of the evolution problem governing the dynamics of a discrete system with one frictional constraint. The status of the constraint function $\varphi$ and of the friction set $\mathcal{C}_{\mathbf{q}}^{*}$ are identical: they are both supposed to be given as part of the modelling process. It will be seen in section 6 , how Coulomb friction of coefficient $\mu$ can be used as a heuristic means to make a systematic proposal for the friction set $\mathcal{C}_{\mathbf{q}}^{*}$, in the most general case.

Before entering that discussion in section 6 , we shall first derive the generalization of problem $\mathcal{P}_{3}^{\prime}$ to the (common) situation where several frictional unilateral constraints must be handled.

\section{The case of one point particle and several frictional unilateral constraints}

The point of view of Lagrange on discrete mechanical system dynamics consists in describing it as the dynamics of a single point on a configuration manifold endowed with a nontrivial Riemannian structure (that induced by the kinetic scalar product on each tangent space). Therefore, the discussion of the dynamics of a discrete mechanical system with several frictional unilateral constraints will be that of a single point evolving on the configuration manifold and obeying several unilateral constraints. It is therefore natural to begin the analysis with the discussion of an academic situation: that of a point particle of unit mass evolving in the usual three-dimensional space and obeying two unilateral constraints described by the two functions $\varphi_{1}$ and $\varphi_{2}$. To lighten the writing a little bit, the functions $\varphi_{1}$ and $\varphi_{2}$ will be assumed to be linear:

$$
\varphi_{\alpha}(\mathbf{q})=\left\langle\mathbf{n}_{\alpha}^{*}, \mathbf{q}\right\rangle, \quad \text { with: }\left\|\mathbf{n}_{\alpha}^{*}\right\|=1,
$$

but this is no restriction of generality. To make the two unilateral constraints distinct, one must assume that $\mathbf{n}_{1}^{*}$ and $\mathbf{n}_{2}^{*}$ are linearly independent and this is nothing but the usual linear independence of the constraints which was already invoked in section 1.3. Coulomb friction with coefficient $\mu_{\alpha}$ is supposed to be associated with the obstacle described by the function $\varphi_{\alpha}$ and the objective is now to design a consistent formulation of the dynamical evolution problem, encompassing the episodes of smooth motion and the impacts. The issue is naturally that of adapting the expression of the friction law and that of the impact law to cases where both unilateral constraints are simultaneously active.

The very first remark is that the definition of the normal and tangential reaction force has to be refined. Indeed, in view of the formulation of the friction law in section 2.2 , it would be natural, in the case where the particle is located at some point of the line $\varphi_{1}(\mathbf{q})=\varphi_{2}(\mathbf{q})=0$, to define the tangential component of the reaction force as its component along that line and the normal component as the component in the orthogonal plane. However, considering the equilibrium of the particle at some point of the line under given external forces, there may exist a force due to friction which is not necessarily directed along the line (think of an equilibrium located on the line, involving friction and an external force which is not directed along the line). This remark leads to define the tangential component of the reaction force as the projection of the reaction force onto the opposite of the tangential cone to the admissible region (that is, the set of vectors $\mathbf{- v}$ where $\mathbf{v}$ belongs to the tangential cone). This projection 
will be denoted by $\mathbf{r}_{\tau}$. The definition will make use of the set $J(\mathbf{q})$ of all the active constraints at the location q:

$$
J(\mathbf{q})=\left\{\alpha \mid \varphi_{\alpha}(\mathbf{q})=0\right\}
$$

and runs as follows. Any vector $\mathbf{r}$ based at the location $\mathbf{q}$ can be uniquely split into the form:

$$
\mathbf{r}=\mathbf{r}_{\tau}+\sum_{\alpha \in J(\mathbf{q})} \lambda_{\alpha} \mathbf{n}_{\alpha}^{*}
$$

with:

$$
\left\langle\sum_{\alpha \in J(\mathbf{q})} \lambda_{\alpha} \mathbf{n}_{\alpha}^{*}, \mathbf{r}_{\tau}\right\rangle=0, \quad \text { and } \forall \alpha \in J(\mathbf{q}), \quad \lambda_{\alpha} \in \mathbb{R}^{+}, \quad\left\langle\mathbf{n}_{\alpha}^{*}, \mathbf{r}_{\tau}\right\rangle \leq 0 .
$$

The second part of that decomposition will be called 'the normal part' and the following notation:

$$
\mathbf{r}_{\nu}=\sum_{\alpha \in J(\mathbf{q})} \lambda_{\alpha} \mathbf{n}_{\alpha}^{*}
$$

will sometimes be used. It is nothing but the projection of $\mathbf{r}$ onto the opposite of the normal cone at location $\mathbf{q}$ to the admissible region defined by the unilateral constraints. With this definition, one can satisfactorily account for all equilibria of the particle at some point of the line $\varphi_{1}(\mathbf{q})=\varphi_{2}(\mathbf{q})=0$, under a given external force, by taking the reaction force in the friction cone defined by:

$$
\mathcal{K}_{\text {frict }} \stackrel{\text { def }}{=}\left\{\mathbf{r}=\mathbf{r}_{\tau}+\sum_{\alpha \in J(\mathbf{q})} \lambda_{\alpha} \mathbf{n}_{\alpha}^{*} \mid \forall \hat{\mathbf{v}} \in \mathbb{R}^{3}, \quad\left\langle\mathbf{r}_{\tau}, \hat{\mathbf{v}}\right\rangle+\sum_{\alpha \in J(\mathbf{q})} \lambda_{\alpha} S_{\mu_{\alpha} \mathbf{B}_{\alpha}^{*}}(-\hat{\mathbf{v}}) \geq 0\right\},
$$

where $\mathbf{B}_{\alpha}^{*}$ denotes the closed unit disk on the plane $\varphi_{\alpha}(\mathbf{q})=0$. Since the functions $S_{\mu_{\alpha} \mathbf{B}_{\alpha}^{*}}$ take only nonnegative values, note that:

$$
\mathbf{r}_{\tau}+\sum_{\alpha \in J(\mathbf{q})} \lambda_{\alpha} \mathbf{n}_{\alpha}^{*}=\mathbf{r} \in \mathcal{K}_{\text {frict }} \quad \Longrightarrow \quad \forall \alpha \in J(\mathbf{q}), \quad \lambda_{\alpha} \in \mathbb{R}^{+}
$$

Let us point out that we introduced a single generalized reaction force to account for several constraints, in accordance with what was done in the frictionless case (section 1.3).

In view of the friction law written in section 2.2 for one unilateral constraint, one now would naturally postulate the friction law for several unilateral constraints in the form:

$$
\forall \hat{\mathbf{v}} \in C^{0}\left([0, T] ; \mathbb{R}^{3}\right),
$$

$$
\int_{[0, T]}\left\langle\mathbf{r}_{\tau}, \hat{\mathbf{v}}-\dot{\mathbf{q}}^{+}\right\rangle+\sum_{\alpha \in J(\mathbf{q})} \lambda_{\alpha}\left[S_{\mu_{\alpha} \mathbf{B}_{\alpha}^{*}}(-\hat{\mathbf{v}})-S_{\mu_{\alpha} \mathbf{B}_{\alpha}^{*}}\left(-\dot{\mathbf{q}}^{+}\right)\right] \geq 0,
$$

which is driven by the principle of adding the dissipations associated with each constraint. But, this last form turns out to be inappropriate for impacts. Indeed, consider an impact within the plane orthogonal to the line $\varphi_{1}(\mathbf{q})=\varphi_{2}(\mathbf{q})=0$, with the particle arriving along the median line between the two lines defined by the obstacles and bouncing along this median line. The reaction force $\dot{\mathbf{q}}^{+}-\dot{\mathbf{q}}^{-}$is along the median line. Therefore, the tangential component $\mathbf{r}_{\tau}$ of the reaction force vanishes. Making the choice $\hat{\mathbf{v}} \equiv \mathbf{0}$ as a test function in the above proposal for the friction law yields a contradiction. Hence, we are led to postulate the friction law under the form:

$$
\forall \hat{\mathbf{v}} \in C^{0}\left([0, T] ; \mathbb{R}^{3}\right),
$$

$$
\int_{[0, T]}\left\langle\mathbf{r}_{\tau}, \hat{\mathbf{v}}-\dot{\mathbf{q}}_{\mathrm{t}}^{+}\right\rangle+\sum_{\alpha \in J(\mathbf{q})} \lambda_{\alpha}\left[S_{\mu_{\alpha} \mathbf{B}_{\alpha}^{*}}(-\hat{\mathbf{v}})-S_{\mu_{\alpha} \mathbf{B}_{\alpha}^{*}}\left(-\dot{\mathbf{q}}_{\mathrm{t}}^{+}\right)\right] \geq 0,
$$

where $\dot{\mathbf{q}}_{\mathrm{t}}^{+}$is the component of the right-velocity along the line $\varphi_{1}(\mathbf{q})=\varphi_{2}(\mathbf{q})=0$. Therefore, the normal/tangential components for velocities should be defined as follows. Any vector $\mathbf{v}$ based at the location $\mathbf{q}$ can be uniquely split into the form:

$$
\mathbf{v}=\mathbf{v}_{\mathrm{t}}+\mathbf{v}_{\mathrm{n}}
$$


with:

$$
\mathbf{v}_{\mathrm{n}} \in \sum_{\alpha \in J(\mathbf{q})} \mathbb{R} \mathbf{n}_{\alpha}^{*}, \quad \text { and } \quad \forall \alpha \in J(\mathbf{q}), \quad\left\langle\mathbf{n}_{\alpha}^{*}, \mathbf{v}_{\mathrm{t}}\right\rangle=0 .
$$

Note that the replacement of $\dot{\mathbf{q}}^{+}$by $\dot{\mathbf{q}}_{\mathrm{t}}^{+}$in the friction law (4.3) makes no difference during a smooth episode of motion. Note also that implementing this replacement in the friction law in problem $\mathcal{P}_{3}^{\prime}$ would make no difference either. Finally, the test functions are still allowed to take values in the whole space in consistency with the definition of $\mathcal{K}_{\text {frict }}$.

For motion with nonvanishing velocity along the line $\varphi_{1}(\mathbf{q})=\varphi_{2}(\mathbf{q})=0$, the friction law (4.3) implements the idea that the frictional dissipation is the sum of the dissipations associated with the friction with each obstacle. For an equilibrium on that line involving the friction force, it is crucial to admit a tangential reaction force which is not necessarily directed along the line, but possibly along anyone of the two hyperplanes. This is the reason why it is necessary to define the normal and tangential parts of the reaction force as a decomposition with respect to the opposite of the normal and tangential cones (formula (4.1)) and not as a decomposition with respect to the normal and tangential vector spaces. For a smooth motion leaving the line but remaining on one of the two obstacles, one recovers the usual Coulomb friction.

The generalized friction law (4.3) is also able to deal with occurrences of impact, that is, instants where the measure $\mathbf{r}$ has a Dirac mass. However, the generalized friction law (4.3) applied during an isolated impact handles only a frictional part and is unable alone to predict the outcome of an impact (as is seen in the frictionless case). It must therefore be complemented with an appropriate impact law. This impact law should be designed to be always compatible with the friction law (4.3) and to deliver exactly the lacking information so that, for any given impacting velocity $\dot{\mathbf{q}}^{-}$, the outcoming velocity is uniquely determined. It is sufficient to ask that the impact law should deliver only the normal component of the right-velocity:

$$
\dot{\mathbf{q}}_{\mathrm{n}}^{+}=\mathcal{F}\left(\mathbf{q}, \dot{\mathbf{q}}^{-}\right),
$$

where the given function $\mathcal{F}$ should fulfill the four following requirements:

- $\forall \mathbf{q}, \dot{\mathbf{q}}^{-}, \quad \mathcal{F}\left(\mathbf{q}, \dot{\mathbf{q}}^{-}\right) \in \sum_{\alpha \in J(\mathbf{q})} \mathbb{R} \mathbf{n}_{\alpha}^{*}$,

$(\mathcal{F}$ delivers normal velocities only),

- $\forall \mathbf{q}, \dot{\mathbf{q}}^{-}, \quad \forall \alpha \in J(\mathbf{q}), \quad\left\langle\mathbf{n}_{\alpha}^{*}, \mathcal{F}\left(\mathbf{q}, \dot{\mathbf{q}}^{-}\right)\right\rangle \geq 0$, $(\mathcal{F}$ delivers velocities which are compatible with the unilateral constraints only),

- $\forall \mathbf{q}, \dot{\mathbf{q}}^{-}, \quad \mathcal{F}\left(\mathbf{q}, \dot{\mathbf{q}}^{-}\right)-\dot{\mathbf{q}}_{\mathrm{n}}^{-} \in \mathcal{K}_{\text {frict }}$,

(the percussion associated with the normal parts of the left and right-velocities must belong to the friction cone). It should be mentioned here, that, if the angle between the two plane obstacles is acute then this percussion has zero tangential component and the condition is automatically fulfilled. But, if the angle is obtuse, then the tangential component of the percussion in the plane orthogonal to the line $\varphi_{1}(\mathbf{q})=\varphi_{2}(\mathbf{q})=0$, can be nonzero and this compatibility condition of the impact law with the friction law must be prescribed.

- $\forall \mathbf{q}, \dot{\mathbf{q}}^{-}, \quad\left\|\mathcal{F}\left(\mathbf{q}, \dot{\mathbf{q}}^{-}\right)\right\|_{\mathbf{q}} \leq\left\|\dot{\mathbf{q}}_{\mathrm{n}}^{-}\right\|_{\mathbf{q}}$,

(there is no increase in kinetic energy due to the impact law).

Note that these requirement are satisfied, in particular, by all the impact laws used in the frictionless case (they can therefore be used in the frictional case). Then, it is an easy exercise to check that for an arbitrary impact, the normal right-velocity being prescribed by the above impact law, the tangential right-velocity is uniquely determined by the friction law (4.3), and that the total right-velocity has a kinetic energy which is never larger than that of the total left-velocity.

Putting all together, we can now write the evolution problem that governs the dynamics of a point particle with several unilateral frictional constraints.

Problem $\mathcal{P}_{4}$. Find $\mathbf{q} \in \operatorname{MMA}\left([0, T] ; \mathbb{R}^{d}\right), \mathbf{r} \in \mathcal{M}\left([0, T] ; \mathbb{R}^{d}\right), \lambda_{\alpha} \in \mathcal{M}([0, T] ; \mathbb{R})$ such that: 
- $\mathbf{q}(0)=\mathbf{q}_{0}, \quad \dot{\mathbf{q}}(0)=\mathbf{v}_{0} \quad$ (initial condition),

- $\ddot{\mathbf{q}}=\mathbf{f}(t ; \mathbf{q}, \dot{\mathbf{q}})+\mathbf{r} \quad$ (equation of motion, in the sense of distributions),

- $\dot{\mathbf{q}}^{+}=\dot{\mathbf{q}}_{\mathrm{n}}^{+}+\dot{\mathbf{q}}_{\mathrm{t}}^{+}, \quad$ with: $\dot{\mathbf{q}}_{\mathrm{n}}^{+} \in \sum_{\alpha \in J(\mathbf{q})} \mathbb{R} \nabla \varphi_{\alpha, \mathbf{q}}, \quad$ and $\forall \alpha \in J(\mathbf{q}), \quad\left\langle\mathrm{d} \varphi_{\alpha, \mathbf{q}}, \dot{\mathbf{q}}_{\mathrm{t}}^{+}\right\rangle=0$,

(split of the right-velocity into normal and tangential components),

- $\mathbf{r}=\mathbf{r}_{\tau}+\sum_{\alpha \in J(\mathbf{q})} \lambda_{\alpha} \mathrm{d} \varphi_{\alpha, \mathbf{q}}, \quad$ with: $\left\langle\sum_{\alpha \in J(\mathbf{q})} \lambda_{\alpha} \nabla \varphi_{\alpha, \mathbf{q}}, \mathbf{r}_{\tau}\right\rangle=0$,

and $\forall \alpha \in J(\mathbf{q}), \quad \lambda_{\alpha} \geq 0, \quad\left\langle\nabla \varphi_{\alpha, \mathbf{q}}, \mathbf{r}_{\tau}\right\rangle \leq 0$,

(split of the reaction force into normal and tangential components),

- $\forall \alpha, \quad \varphi_{\alpha}(\mathbf{q}) \geq 0, \quad \lambda_{\alpha} \varphi_{\alpha}(\mathbf{q}) \equiv 0 \quad$ (contact conditions),

- $\forall \hat{\mathbf{v}} \in C^{0}\left([0, T] ; \mathbb{R}^{3}\right)$,

$\int_{[0, T]}\left\langle\mathbf{r}_{\tau}, \hat{\mathbf{v}}-\dot{\mathbf{q}}_{\mathbf{t}}^{+}\right\rangle+\sum_{\alpha \in J(\mathbf{q})} \lambda_{\alpha}\left[S_{\mu_{\alpha} \mathbf{B}_{\alpha}^{*}}(-\hat{\mathbf{v}})-S_{\mu_{\alpha} \mathbf{B}_{\alpha}^{*}}\left(-\dot{\mathbf{q}}_{\mathbf{t}}^{+}\right)\right] \geq 0$,

(Coulomb friction),

- $\dot{\mathbf{q}}_{\mathrm{n}}^{+}=\mathcal{F}\left(\mathbf{q}, \dot{\mathbf{q}}^{-}\right), \quad$ (impact constitutive equation),

where $\mathcal{F}$ is a given function satisfying the above four consistency requirements. These requirements ensure the consistency of the formulation, and in particular, the fact that the outcome of any impact is uniquely determined and energetically consistent. However, it should be mentioned that within this framework, the percussion reaction force $r$ is allowed to be such that $\left\langle\mathbf{r}_{\tau}, \dot{\mathbf{q}}^{+}\right\rangle>0$, which should probably be precluded in a practical proposal of an impact law $\mathcal{F}$.

\section{The case of a discrete mechanical system with several frictional unilateral constraints}

It is now an easy matter to combine the results of sections 3 and 4 to reach the objective of the article: stating the general evolution problem governing the dynamics of discrete mechanical systems with several frictional unilateral constraints in a mathematically consistent way. The only differences with the situation analyzed in the previous section is that the mass matrix $\mathbf{M}(\mathbf{q})$ no longer needs to be proportional to the identity (which implies that $\mathrm{d} \varphi_{\alpha}$ and $\nabla \varphi_{\alpha}$ must be carefully distinguished) and that the $\mathcal{C}_{\alpha, \mathbf{q}}^{*}$ no longer need to be disks and, in particular, they may be unbounded sets.

To sum up, we are given a discrete mechanical system with configuration manifold $Q$ of finite dimension $d$. We are also given the kinetic matrix $\mathbf{M}(\mathbf{q})$ supposed to be symmetric and positive definite so that it defines an (intrinsic) scalar product on each tangent space $T_{\mathbf{q}} Q$ to the configuration manifold (which is nothing but the $d$-dimensional space of all the virtual generalized velocities in the configuration $\mathbf{q})$. Some generalized forces $\mathbf{f}(t ; \mathbf{q}, \dot{\mathbf{q}})$ are also given depending on the time $t$ but also possibly on the current configuration and velocity of the system. In addition, $n$ unilateral constraints are described by a given collection of smooth functions $\varphi_{\alpha}: Q \rightarrow \mathbb{R}$, supposed to satisfy the following independence condition:

the $\mathrm{d} \varphi_{\alpha, \mathbf{q}}(\alpha \in J(\mathbf{q}))$ are linearly independent, where we recall the notation $J(\mathbf{q})=\{\alpha \mid$ $\left.\varphi_{\alpha}(\mathbf{q})=0\right\}$.

With each of these constraints is associated a set $\mathcal{C}_{\alpha, \mathbf{q}}^{*}$ describing the dry friction attached to the unilateral constraint. The set $\mathcal{C}_{\alpha, \mathbf{q}}^{*}$ is supposed to be a nonempty closed convex subset of $T_{\mathbf{q}}^{*} Q$, which is contained in the hyperplane:

$$
\mathcal{T}_{\alpha, \mathbf{q}}^{*}=\left\{\mathbf{f} \in T_{\mathbf{q}}^{*} Q \mid \mathbf{f} \cdot \mathbf{M}^{-1}(\mathbf{q}) \cdot \mathrm{d} \varphi_{\alpha, \mathbf{q}}=0\right\} .
$$

Finally, the general evolution problem that governs the dynamics of the discrete mechanical system with these $n$ unilateral frictional constraints reads as:

Problem $\mathcal{P}_{4}^{\prime}$. Find $\mathbf{q} \in M M A([0, T] ; Q), \mathbf{r} \in \mathcal{M}\left([0, T] ; T^{*} Q\right), \lambda_{\alpha} \in \mathcal{M}([0, T] ; \mathbb{R})$ such that: 
- $\mathbf{q}(0)=\mathbf{q}_{0}, \quad \dot{\mathbf{q}}(0)=\mathbf{v}_{0} \quad$ (initial condition),

- $\mathbf{M}(\mathbf{q}) \cdot \frac{\mathrm{D} \dot{\mathbf{q}}}{\mathrm{D} t}=\mathbf{f}\left(t ; \mathbf{q}, \dot{\mathbf{q}}^{-}\right)+\mathbf{r} \quad$ (equation of motion, in the sense of distributions),

- $\dot{\mathbf{q}}^{+}=\dot{\mathbf{q}}_{\mathrm{n}}^{+}+\dot{\mathbf{q}}_{\mathrm{t}}^{+}, \quad$ with: $\dot{\mathbf{q}}_{\mathrm{n}}^{+} \in \sum_{\alpha \in J(\mathbf{q})} \mathbb{R} \nabla \varphi_{\alpha, \mathbf{q}}, \quad$ and $\forall \alpha \in J(\mathbf{q}), \quad\left\langle\mathrm{d} \varphi_{\alpha, \mathbf{q}}, \dot{\mathbf{q}}_{\mathrm{t}}^{+}\right\rangle=0$,

(split of the right-velocity into normal and tangential components),

- $\mathbf{r}=\mathbf{r}_{\tau}+\sum_{\alpha \in J(\mathbf{q})} \lambda_{\alpha} \mathrm{d} \varphi_{\alpha, \mathbf{q}}, \quad$ with: $\left\langle\sum_{\alpha \in J(\mathbf{q})} \lambda_{\alpha} \nabla \varphi_{\alpha, \mathbf{q}}, \mathbf{r}_{\tau}\right\rangle=0$,

and $\forall \alpha \in J(\mathbf{q}), \quad \lambda_{\alpha} \geq 0, \quad\left\langle\nabla \varphi_{\alpha, \mathbf{q}}, \mathbf{r}_{\tau}\right\rangle \leq 0$,

(split of the reaction force into normal and tangential components),

- $\forall \alpha, \quad \varphi_{\alpha}(\mathbf{q}) \geq 0, \quad \lambda_{\alpha} \varphi_{\alpha}(\mathbf{q}) \equiv 0 \quad$ (contact conditions),

- $\forall \hat{\mathbf{v}} \in C^{0}\left([0, T] ; T_{\mathbf{q}} Q\right)$,

$\int_{[0, T]}\left\langle\mathbf{r}_{\tau}, \hat{\mathbf{v}}-\dot{\mathbf{q}}_{\mathrm{t}}^{+}\right\rangle+\sum_{\alpha \in J(\mathbf{q})} \lambda_{\alpha}\left[S_{\mathcal{C}_{\alpha, \mathbf{q}}^{*}}(-\hat{\mathbf{v}})-S_{\mathcal{C}_{\alpha, \mathbf{q}}^{*}}\left(-\dot{\mathbf{q}}_{\mathrm{t}}^{+}\right)\right] \geq 0$

(generalized friction law),

- $\dot{\mathbf{q}}_{\mathrm{n}}^{+}=\mathcal{F}\left(\mathbf{q}, \dot{\mathbf{q}}^{-}\right), \quad$ (impact constitutive equation),

where the given function $\mathcal{F}$ is required to fulfill the four following consistency conditions:

- $\forall \mathbf{q}, \dot{\mathbf{q}}^{-}, \quad \mathcal{F}\left(\mathbf{q}, \dot{\mathbf{q}}^{-}\right) \in \sum_{\alpha \in J(\mathbf{q})} \mathbb{R} \mathbf{n}_{\alpha}^{*}$,

$(\mathcal{F}$ delivers normal velocities only),

- $\forall \mathbf{q}, \dot{\mathbf{q}}^{-}, \quad \forall \alpha \in J(\mathbf{q}), \quad\left\langle\mathbf{n}_{\alpha}^{*}, \mathcal{F}\left(\mathbf{q}, \dot{\mathbf{q}}^{-}\right)\right\rangle \geq 0$, $\left\langle\mathbf{n}_{\alpha}^{*}, \mathcal{F}\left(\mathbf{q}, \dot{\mathbf{q}}^{-}\right)\right\rangle=0 \quad \Longrightarrow \quad \mathcal{F}\left(\mathbf{q}, \dot{\mathbf{q}}^{-}\right) \in D\left(S_{\mathcal{C}_{\alpha, \mathbf{q}}^{*}}\right)$,

$(\mathcal{F}$ delivers velocities which are compatible with the unilateral constraints only and with the possible unbounded $\mathcal{C}_{\alpha, \mathbf{q}}^{*}$ which forbid certain values of right-velocities),

- $\forall \mathbf{q}, \dot{\mathbf{q}}^{-}, \quad \mathbf{M}(q) \cdot\left(\mathcal{F}\left(\mathbf{q}, \dot{\mathbf{q}}^{-}\right)-\dot{\mathbf{q}}_{\mathrm{n}}^{-}\right) \in \mathcal{K}_{\text {frict }}$,

where the friction cone at location $\mathbf{q}$ is defined as:

$\mathcal{K}_{\text {frict }} \stackrel{\text { def }}{=}\left\{\mathbf{r}=\mathbf{r}_{\tau}+\sum_{\alpha \in J(\mathbf{q})} \lambda_{\alpha} \mathbf{n}_{\alpha}^{*} \mid \forall \hat{\mathbf{v}} \in T_{\mathbf{q}} Q, \quad\left\langle\mathbf{r}_{\tau}, \hat{\mathbf{v}}\right\rangle+\sum_{\alpha \in J(\mathbf{q})} \lambda_{\alpha} S_{\mathcal{C}_{\alpha, \mathbf{q}}^{*}}(-\hat{\mathbf{v}}) \geq 0\right\}$,

(the percussion associated with the normal components of the left and right-velocities must belong to the friction cone).

- $\forall \mathbf{q}, \dot{\mathbf{q}}^{-}, \quad\left\|\mathcal{F}\left(\mathbf{q}, \dot{\mathbf{q}}^{-}\right)\right\|_{\mathbf{q}} \leq\left\|\dot{\mathbf{q}}_{\mathrm{n}}^{-}\right\|_{\mathbf{q}}$,

(there is no increase in kinetic energy due to the impact law).

We claim that problem $\mathcal{P}_{4}^{\prime}$ above is the first consistent general formulation of the dynamics of discrete mechanical systems with several frictional unilateral constraints. We now enumerate some comments about problem $\mathcal{P}_{4}^{\prime}$.

1. The impact law supplies only the normal component $\dot{\mathbf{q}}_{n}^{+}$of the outcoming velocity. It has the same form as in the frictionless case. The consistency requirements render the impact law compatible with the unilateral constraints and the friction law. In the case where $J(\mathbf{q})$ reduces to a single element (only one active constraint), these requirements entail that the impact law reduces to:

$$
\dot{q}_{\mathrm{n}}^{+}=-e\left(\mathbf{q}, \dot{\mathbf{q}}^{-}\right) \dot{q}_{\mathrm{n}}^{-},
$$

where the restitution coefficient $e\left(\mathbf{q}, \dot{\mathbf{q}}^{-}\right) \in[0,1]$. 
2. The tangential component of the velocity is handled by the friction law which governs friction indifferently during the smooth phases of the motion and the impacts. In the particular case where the system is a collection of point particles and the data are analytic functions, it was proved in [5] that the corresponding evolution problem has a unique solution. This entails in particular that the tangential component $\dot{\mathbf{q}}_{t}^{+}$of the outcoming velocity at an impact instant is uniquely determined by the incoming velocity $\dot{\mathbf{q}}^{-}$.

3. In addition, proposition 5.1 below shows that the dissipativity (energetic consistency) of any solution of problem $\mathcal{P}_{4}^{\prime}$ is automatically ensured, that is, energetic consistency is built-in within the formulation.

Proposition 5.1. Let $\mathbf{q} \in \operatorname{MMA}([0, T] ; Q), \mathbf{r} \in \mathcal{M}\left([0, T] ; T^{*} Q\right)$ be a solution of problem $\mathcal{P}_{4}^{\prime}$. Then, it is dissipative in the sense that for all $t_{1}, t_{2} \in[0, T]$, such that $t_{1} \leq t_{2}$, one has:

$$
\frac{1}{2} \dot{\mathbf{q}}^{+}\left(t_{2}\right) \cdot \mathbf{M}\left(\mathbf{q}\left(\mathbf{t}_{2}\right)\right) \cdot \dot{\mathbf{q}}^{+}\left(t_{2}\right) \leq \frac{1}{2} \dot{\mathbf{q}}^{-}\left(t_{1}\right) \cdot \mathbf{M}\left(\mathbf{q}\left(\mathbf{t}_{1}\right)\right) \cdot \dot{\mathbf{q}}^{-}\left(t_{1}\right)+\int_{t_{1}}^{t_{2}}\left\langle\mathbf{f}\left(t ; \mathbf{q}(t), \dot{\mathbf{q}}^{-}(t)\right), \dot{\mathbf{q}}^{-}(t)\right\rangle_{\mathbf{q}} \mathrm{d} t .
$$

Proof. We multiply the equation of motion by $\left(\dot{\mathbf{q}}^{+}(t)+\dot{\mathbf{q}}^{-}(t)\right) / 2$ and integrate over $\left[t_{1}, t_{2}\right]$ (the function $\left.\left(\dot{\mathbf{q}}^{+}(t)+\dot{\mathbf{q}}^{-}(t)\right) / 2\right)$, having bounded variation, is universally integrable). We get:

$$
\begin{aligned}
\frac{1}{2} \dot{\mathbf{q}}^{+}\left(t_{2}\right) \cdot \mathbf{M}\left(\mathbf{q}\left(t_{2}\right)\right) \cdot \dot{\mathbf{q}}^{+}\left(t_{2}\right)-\frac{1}{2} \dot{\mathbf{q}}^{-}\left(t_{1}\right) \cdot \mathbf{M}\left(\mathbf{q}\left(t_{1}\right)\right) \cdot \dot{\mathbf{q}}^{-}\left(t_{1}\right)=\int_{\left[t_{1}, t_{2}\right]} \frac{\dot{\mathbf{q}}^{+}+\dot{\mathbf{q}}^{-}}{2} \cdot \mathbf{M}(\mathbf{q}) \cdot \frac{\mathrm{D} \dot{\mathbf{q}}}{\mathrm{D} t} \\
=\int_{t_{1}}^{t_{2}}\left\langle\mathbf{f}\left(t ; \mathbf{q}(t), \dot{\mathbf{q}}^{-}(t)\right), \dot{\mathbf{q}}^{-}(t)\right\rangle_{\mathbf{q}} \mathrm{d} t+\int_{\left[t_{1}, t_{2}\right]}\left\langle\mathbf{r}, \frac{\dot{\mathbf{q}}^{+}+\dot{\mathbf{q}}^{-}}{2}\right\rangle_{\mathbf{q}}
\end{aligned}
$$

Hence, the proof of proposition 5.1 amounts to proving:

$$
\int_{\left[t_{1}, t_{2}\right]}\left\langle\mathbf{r}, \frac{\dot{\mathbf{q}}^{+}+\dot{\mathbf{q}}^{-}}{2}\right\rangle_{\mathbf{q}} \leq 0 .
$$

Let $\mathcal{I}$ denote the set of all the instants of impact (that is, those $t$ such that $\dot{\mathbf{q}}^{+}(t) \neq \dot{\mathbf{q}}^{-}(t)$ ). Since $\mathbf{q} \in M M A([0, T] ; Q), \dot{\mathbf{q}}^{+}(t)$ and $\dot{\mathbf{q}}^{-}(t)$ are both functions with bounded variation and the set $\mathcal{I}$ is at most countable. We have:

$$
\begin{aligned}
\int_{\left[t_{1}, t_{2}\right]}\left\langle\mathbf{r}, \frac{\dot{\mathbf{q}}^{+}+\dot{\mathbf{q}}^{-}}{2}\right\rangle_{\mathbf{q}}= & \int_{\mathcal{I}}\left\langle\mathbf{r}, \frac{\dot{\mathbf{q}}^{+}+\dot{\mathbf{q}}^{-}}{2}\right\rangle_{\mathbf{q}} \\
& +\int_{\left[t_{1}, t_{2}\right] \backslash \mathcal{I}}\left\langle\sum_{\alpha \in J(\mathbf{q})} \lambda_{\alpha} \mathrm{d} \varphi_{\alpha, \mathbf{q}}, \frac{\dot{\mathbf{q}}^{+}+\dot{\mathbf{q}}^{-}}{2}\right\rangle_{\mathbf{q}}+\int_{\left[t_{1}, t_{2}\right] \backslash \mathcal{I}}\left\langle\mathbf{r}_{\tau}, \frac{\dot{\mathbf{q}}^{+}+\dot{\mathbf{q}}^{-}}{2}\right\rangle_{\mathbf{q}} .
\end{aligned}
$$

Note that:

$$
\forall t \in\left[t_{1}, t_{2}\right], \quad \forall \alpha \in J(\mathbf{q}(t)), \quad\left\langle\mathrm{d} \varphi_{\alpha, \mathbf{q}}, \dot{\mathbf{q}}^{+}\right\rangle_{\mathbf{q}} \geq 0, \quad\left\langle\mathrm{~d} \varphi_{\alpha, \mathbf{q}}, \dot{\mathbf{q}}^{-}\right\rangle_{\mathbf{q}} \leq 0 .
$$

Since $\dot{\mathbf{q}}^{+}=\dot{\mathbf{q}}^{-}$on $\left[t_{1}, t_{2}\right] \backslash \mathcal{I}, \dot{\mathbf{q}}^{+}$and $\dot{\mathbf{q}}^{-}$reduce to their tangential component and the third integral in the right handside of (5.1) must vanish. Also, the friction law implies:

$$
\forall \hat{\mathbf{v}} \in C^{0}\left(\left[t_{1}, t_{2}\right] ; T_{\mathbf{q}} Q\right), \quad \int_{\left[t_{1}, t_{2}\right]}\left\langle\mathbf{r}_{\tau}, \hat{\mathbf{v}}-\dot{\mathbf{q}}_{\mathrm{t}}^{+}\right\rangle_{\mathbf{q}}+\sum_{\alpha \in J(\mathbf{q})} \lambda_{\alpha}\left[S_{\mathcal{C}_{\alpha, \mathbf{q}}^{*}}(-\hat{\mathbf{v}})-S_{\mathcal{C}_{\alpha, \mathbf{q}}^{*}}\left(-\dot{\mathbf{q}}_{\mathrm{t}}^{+}\right)\right] \geq 0 .
$$

Choosing the identically vanishing function $\hat{\mathbf{v}}$ and using the fact that each function $S_{\mathcal{C}_{\alpha, \mathbf{q}}^{*}}$ is nonnegative (because $\mathbf{0} \in \mathcal{C}_{\alpha, \mathbf{q}}^{*}$ ), we get:

$$
\int_{\left[t_{1}, t_{2}\right]}\left\langle\mathbf{r}_{\tau}, \dot{\mathbf{q}}_{\mathrm{t}}^{+}\right\rangle_{\mathbf{q}} \leq 0
$$


Since this is true for all $t_{1} \leq t_{2}$, this entails that $\left\langle\mathbf{r}_{\tau}, \dot{\mathbf{q}}_{\mathbf{t}}^{+}\right\rangle_{\mathbf{q}}$ is a nonpositive measure and:

$$
\int_{\left[t_{1}, t_{2}\right] \backslash \mathcal{I}}\left\langle\mathbf{r}_{\tau}, \frac{\dot{\mathbf{q}}^{+}+\dot{\mathbf{q}}^{-}}{2}\right\rangle_{\mathbf{q}}=\int_{\left[t_{1}, t_{2}\right] \backslash \mathcal{I}}\left\langle\mathbf{r}_{\tau}, \dot{\mathbf{q}}_{\mathrm{t}}^{+}\right\rangle_{\mathbf{q}} \leq 0 .
$$

Hence, (5.1) reduces to:

$$
\int_{\left[t_{1}, t_{2}\right]}\left\langle\mathbf{r}, \frac{\dot{\mathbf{q}}^{+}+\dot{\mathbf{q}}^{-}}{2}\right\rangle_{\mathbf{q}} \leq \int_{\mathcal{I}}\left\langle\mathbf{r}, \frac{\dot{\mathbf{q}}^{+}+\dot{\mathbf{q}}^{-}}{2}\right\rangle_{\mathbf{q}} .
$$

But, by the equation of motion, the generalized reaction force measure $\mathbf{r}$ has an atom of amplitude $\left.\mathbf{M}(\mathbf{q}(t)) \cdot\left(\dot{\mathbf{q}}^{+} t\right)-\dot{\mathbf{q}}^{-}(t)\right)$ at each $t \in \mathcal{I}$. Therefore, using the fact that $\mathcal{I}$ is countable:

$$
\int_{\left[t_{1}, t_{2}\right]}\left\langle\mathbf{r}, \frac{\dot{\mathbf{q}}^{+}+\dot{\mathbf{q}}^{-}}{2}\right\rangle_{\mathbf{q}} \leq \int_{\mathcal{I}}\left\langle\mathbf{r}, \frac{\dot{\mathbf{q}}^{+}+\dot{\mathbf{q}}^{-}}{2}\right\rangle_{\mathbf{q}}=\sum_{t \in \mathcal{I}} \frac{\left\|\dot{\mathbf{q}}_{\mathrm{n}}^{+}\right\|_{\mathbf{q}}^{2}-\left\|\dot{\mathbf{q}}_{\mathrm{n}}^{-}\right\|_{\mathbf{q}}^{2}}{2}+\sum_{t \in \mathcal{I}} \frac{\left\|\dot{\mathbf{q}}_{\mathrm{t}}^{+}\right\|_{\mathbf{q}}^{2}-\left\|\dot{\mathbf{q}}_{\mathrm{t}}^{-}\right\|_{\mathbf{q}}^{2}}{2}
$$

Here, the first sum is nonpositive, thanks to the energetic consistency requirement on the impact law:

$$
\left\|\dot{\mathbf{q}}_{\mathrm{n}}^{+}\right\|_{\mathbf{q}}^{2}=\left\|\mathcal{F}\left(\mathbf{q}, \dot{\mathbf{q}}^{-}\right)\right\|_{\mathbf{q}}^{2} \leq\left\|\dot{\mathbf{q}}_{\mathrm{n}}^{-}\right\|_{\mathbf{q}}^{2} \text {. }
$$

So, it is now sufficient to prove that the second sum is also nonpositive to achieve the proof of the proposition. Since the measure $\left\langle\mathbf{r}_{\tau}, \dot{\mathbf{q}}_{\mathrm{t}}^{+}\right\rangle_{\mathbf{q}}$ is nonpositive, we get:

$$
\forall t \in \mathcal{I}, \quad\left(\dot{\mathbf{q}}_{\mathrm{t}}^{+}-\dot{\mathbf{q}}_{\mathrm{t}}^{-}, \dot{\mathbf{q}}_{\mathrm{t}}^{+}\right)_{\mathbf{q}} \leq 0 .
$$

But, by Cauchy-Schwarz inequality, this entails:

$$
\left\|\dot{\mathbf{q}}_{\mathrm{t}}^{+}\right\|_{\mathbf{q}} \leq\left\|\dot{\mathbf{q}}_{\mathrm{t}}^{-}\right\|_{\mathbf{q}}
$$

and, therefore, the desired conclusion.

\section{Discussion}

Problem $\mathcal{P}_{4}^{\prime}$ is the general form that takes the evolution problem raised by frictional unilateral multibody dynamics when rationally inserted into Lagrange's general framework for the dynamics of systems of rigid bodies. The former formulations that can be found in the literature were all based on the paradigm of Coulomb friction applying on reaction forces of the real world and were actually only available for:

- systems of point particles (see for example [14] or [5])

- very simple systems of rigid bodies with most often only one contact point, because in that case reaction in the real world can be expressed in terms of the generalized reaction force (see for example [26]).

In this section, the status of the data in problem $\mathcal{P}_{4}^{\prime}$ will be discussed, and it will be shown how the paradigm of pointwise Coulomb friction can be used as a heuristic method to propose a systematic construction of the friction sets $\mathcal{C}_{\alpha, \mathbf{q}}^{*}$ (subsection 6.1). Then, simple examples will be considered in order to discuss how problem $\mathcal{P}_{4}^{\prime}$ compares with usual approaches in the field, when applicable.

\subsection{A general method of construction of the friction sets $\mathcal{C}_{\alpha, q}^{*}$}

The general form of the evolution problem raised by frictional unilateral multibody dynamics (problem $\mathcal{P}_{4}^{\prime}$ ) implements some data namely:

- The initial condition $\left(\mathbf{q}_{0}, \mathbf{v}_{0}\right)$.

- The force mapping $\mathbf{f}(t ; \mathbf{q}, \dot{\mathbf{q}})$.

- The constraint functions $\varphi_{\alpha}$.

- The friction sets $\mathcal{C}_{\alpha, \mathbf{q}}^{*}$.

- The function $\mathcal{F}$ that implements the impact constitutive equation. 
The modelling process consists in translating a practical situation in terms of these data, so that studying that practical situation reduces hereafter in studying the corresponding mathematical evolution problem $\mathcal{P}_{4}^{\prime}$. Identifying the initial condition $\left(\mathbf{q}_{0}, \mathbf{v}_{0}\right)$ and the force mapping $\mathbf{f}(t ; \mathbf{q}, \dot{\mathbf{q}})$ is something usual in smooth (that is, without any unilateral restriction) dynamics, so no further comment will be made on this.

The constraint functions $\varphi_{\alpha}$ and the inequalities they define are the way the conditions like non-interpenetration are expressed mathematically. In frictionless unilateral multibody dynamics, their use is now common. They are needed for theoretical investigations (based on problem $\mathcal{P}_{2}$ ), such as existence and uniqueness analysis of solution for the evolution problem, convergence of numerical methods, stability, control... Yet, their consideration is of poor interest for the purpose of practical computations. For very simple systems, it is generally an easy matter to identify them. But, in the case of a complicated system for which numerical solving is unavoidable, the constraint functions $\varphi_{\alpha}$ will never be expressed at first. An algorithm for detection of collisions will be used instead and the functions $\varphi_{\alpha}$ will be only evaluated (approximated) in the neighbourhood of the current configuration of the system.

The status of the friction sets $\mathcal{C}_{\alpha, \mathbf{q}}^{*}$ is the same as that of the constraint functions $\varphi_{\alpha}$ : they are a mathematical expression of the cone of admissible generalized reaction forces, associated with a given activated constraint $\varphi_{\alpha}$. They have to be postulated, but the paradigm of Coulomb's law applying on forces of the real world can be used conveniently in a systematic way to build a proposal for the $\mathcal{C}_{\alpha, \mathbf{q}}^{*}$ as follows.

Sticking to the notations of section 1 , the real world velocity $\tilde{\mathbf{v}}$ of the material point located at $\mathbf{x}$ is expressed in terms of the generalized velocity $\mathbf{v}$ under the form:

$$
\tilde{\mathbf{v}}(\mathbf{x})=\mathbf{l}(\mathbf{q}, \mathbf{x}) \cdot \mathbf{v}
$$

where $\mathbf{l}(\mathbf{q}, \mathbf{x})$ is a linear mapping which depends on the configuration $\mathbf{q}$. Now, consider the configuration $\mathbf{q}$ supposed to render active the constraint $\varphi_{\alpha}: \varphi_{\alpha}(\mathbf{q})=0$. Suppose in addition that active contact is only fulfilled in finitely many material points $\mathbf{x}_{i}$ (in practice, the $x_{i}$ realizing the contact are provided by the collision detection algorithm which evaluates the constraint function locally). Then, given fictitious real world reaction forces $\tilde{\mathbf{r}}_{i}$ based at $\mathbf{x}_{i}$, the generalized reaction force $\mathbf{r}$ reads as:

$$
\mathbf{r}=\sum_{i}{ }^{\mathrm{t}} \mathbf{l}\left(\mathbf{q}, \mathbf{x}_{i}\right) \cdot \tilde{\mathbf{r}}_{i}
$$

where the finite sum has to be replaced by the appropriate integral with respect to the measure $\tilde{\mathbf{r}}$ in the case where the contact material points do not reduce to a finite set (case of conforming material surfaces). In the case where at least one of the bodies in contact has smooth geometry near the contact points, it is usual to postulate that the real world reaction forces $\tilde{\mathbf{r}}_{i}$ must belong to the Coulomb cone $C_{i}$, that is a cone in the three-dimensional space, pointed at $\{\mathbf{0}\}$ and whose axis of revolution is directed along the normal to the solids in contact. In that case, the generalized reaction force $\mathbf{r}$ must belong to the set:

$$
\sum_{i}{ }^{\mathrm{t}} \mathbf{l}(\mathbf{q}, \mathbf{x}) \cdot C_{i}
$$

which is a closed convex cone, pointed at $\{\mathbf{0}\}$, in the space $T_{\mathbf{q}}^{*} Q$ of all generalized forces. Hence, the paradigm of Coulomb law applying on real world forces offers a systematic heuristic means to make a proposal for the friction set $\mathcal{C}_{\alpha, \mathbf{q}}^{*}$ by taking a section of the above cone:

$$
\mathcal{C}_{\alpha, \mathbf{q}}^{*}=\left\{\mathbf{r}_{\mathrm{t}} \in \mathcal{T}_{\mathbf{q}}^{*} \mid \mathbf{r}_{\mathrm{t}}+\mathbf{n}^{*} \in \sum_{i}{ }^{\mathrm{t}} \mathbf{l}(\mathbf{q}, \mathbf{x}) \cdot C_{i}\right\},
$$

where the notations $\mathbf{n}^{*}$ and $\mathcal{T}_{\mathbf{q}}^{*}$ are those introduced in section 3.1. The set $\mathcal{C}_{\alpha, \mathbf{q}}^{*}$, constructed in such a way, will always be a closed convex subset of $\mathcal{T}_{\mathbf{q}}^{*}$, containing $\mathbf{0}$. It may however be unbounded in some cases (an example will be displayed in the next section), as the cone

$$
\sum_{i}{ }^{\mathrm{t}} \mathbf{l}(\mathbf{q}, \mathbf{x}) \cdot C_{i}
$$


may contain elements $\mathbf{r}$ such that $r_{\mathrm{n}}=\mathbf{r} \cdot \mathbf{M}^{-1}(\mathbf{q}) \cdot \mathbf{n}^{*}<0$.

Once again, the status of the friction sets is exactly the same as that of the constraint functions $\varphi_{\alpha}$ : their existence is postulated in order to perform theoretical investigations of wide significance on frictional unilateral multibody dynamics. The preceding technique provides a systematic way to build a proposal based on the paradigm of Coulomb friction applying on the real world reactions forces. This general technique will be used to form an explicit proposal for the analysis of very simple system, as seen in the example in the next subsection. In case of numerical computation of a complex system, it will only be needed to evaluate the friction $\operatorname{sets} \mathcal{C}_{\alpha, \mathbf{q}}^{*}$ at the configurations $\mathbf{q}$ that are encountered during the computation.

Finally, the impact constitutive equation has the same status as the constitutive equation in continuum mechanics. The function $\mathcal{F}$ must be postulated. Most often, it will be sufficient to consider only impacts where one constraint $\varphi_{\alpha}$ is active (such impacts are often said simple), and only a restitution coefficient for the normal components of generalized velocities will be needed. In the case of impacts that are not necessarily simple, the consistency conditions are meant to guide the proposal of the impact constitutive equation to guarantee its consistency (for example, the impact constitutive equation that produce a velocity after impact which is not compatible with the unilateral constraint should not be allowed.)

\subsection{An example of Painlevé type}

In the case of a system of point particles, the components of the generalized reaction force are also components of the real world reaction forces, and our point of view reduces to the usual one. Differences arise only in the case of a system involving rigid bodies that are not point particles. So, from now on, we focus on such systems involving, in particular, a rigid bar.

The first example that will be considered is similar to the historical example raised by Painlevé [19] and which was the basis of a decades long controversy, about the question 'is Coulomb's friction law inconsistent with rigid body dynamics?'. This example is actually very specific, since it is a case where the real world reaction forces can be recovered from the generalized reaction forces. We point out again that, though the generalized reaction forces can always be calculated from the the real world reaction forces, the reverse calculation is in general not possible. The following example is an example where this reverse calculation is possible, resulting in a strong incentive to use the real world reaction forces to formulate the friction law. This fact gives poor generality to the example, that provokes implicitly the general idea that the friction law could be formulated as easily in terms of the real world reaction forces in more general situations. Subsequent examples will be provided in order to refute this idea.

We consider the simplest two degrees of freedom (d.o.f.) discrete mechanical system with nontrivial mass matrix. An example of such a system is provided by a rigid bar evolving in a plane and whose center of mass is constrained to remain on a line. In the sequel, $G$ will stand for the center of mass and $A$ for one of the two end points of the bar. A convenient parametrization for that system is the pair $(x, \theta)=\mathbf{q}$ where $x$ is the abscissa of $G$ and $\theta$ the angle from $G x$ to the direction of the bar (see figure 1).

The kinetic matrix reads as:

$$
\mathbf{M}(\mathbf{q})=\left(\begin{array}{cc}
m & 0 \\
0 & J
\end{array}\right)
$$

where $m$ is the total mass of the bar and $J$ is the inertia momentum of the bar around the center of mass. Generalized forces $\mathbf{f}$ have two components $f_{x}$ and $f_{\theta}$. Because the considered system is so simple, the two components of the generalized forces can be given a simple mechanical interpretation: they are respectively the $x$-component of the total force and the torque evaluated at the center of mass. Dynamics of this system subjected to external forces $\mathbf{f}$ obeys the equation of motion:

$$
\begin{array}{l|l|l}
\mathbf{M}(\mathbf{q}) \cdot \frac{\mathrm{D} \dot{\mathbf{q}}}{\mathrm{D} t}=\mathbf{f} \quad \Longleftrightarrow \quad & \begin{array}{l}
m \ddot{x}=f_{x}, \\
J \ddot{\theta}=f_{\theta} .
\end{array}
\end{array}
$$




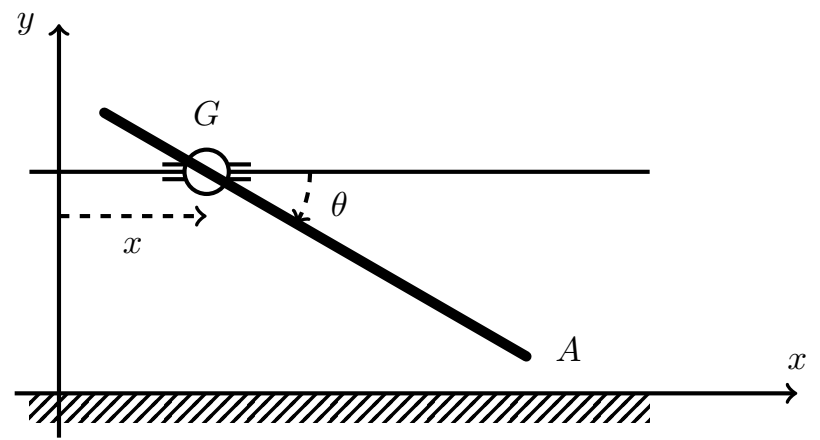

Figure 1. A simple discrete mechanical system with two d.o.f.

Now, it is assumed that the $x$-axis is a rigid obstacle so that the extremity $A$ of the bar must evolve in the half-plane $y \geq 0$. The value of $\theta$, when $A$ lies on the $x$-axis, being denoted by $\theta_{0}$, it is readily checked that this unilateral constraint can be described by:

$$
\varphi(\mathbf{q}) \geq 0, \quad \varphi(x, \theta) \stackrel{\text { def }}{=} \theta-\theta_{0} .
$$

We assume $-\pi / 2<\theta_{0}<0$ as on figure 1 and we restrict our attention to situations where $\theta$ will never exceed $\theta_{0}+\pi$ so that it is not necessary to consider the second unilateral constraint $\pi-\theta_{0}-\theta \geq 0$.

The generalized reaction force is denoted by $\mathbf{r}=\left(r_{x}, r_{\theta}\right)$, and can be expressed in terms of a real world force $\tilde{\mathbf{r}}=\left(\tilde{r}_{x}, \tilde{r}_{y}\right)$ as follows.

$$
\mathbf{r}={ }^{\mathrm{t}} \mathbf{l}(\mathbf{q}, \mathbf{x}) \cdot \tilde{\mathbf{r}} \quad \Longleftrightarrow \quad \begin{aligned}
& r_{x}=\tilde{r}_{x}, \\
& r_{\theta}=l \cos \theta_{0} \tilde{r}_{y}-l \sin \theta_{0} \tilde{r}_{x} .
\end{aligned}
$$

where the fact that $\theta=\theta_{0}$ in any configuration fulfilling the contact condition, has been used. The example under consideration has the exceptional property that the equations (6.1) can be inverted:

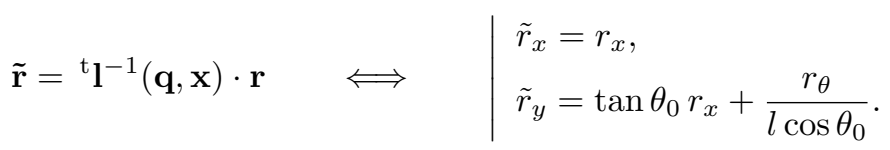

This fact has been an incentive to formulate the evolution problem in terms of the Coulomb friction law applying on the real world reaction force $\tilde{\mathbf{r}}$ since Painlevé. Such a formulation can be found in Stewart [26], in the case of the completely inelastic impact law and a conservative external force $(\mathbf{f}=\mathrm{d} V(\mathbf{q}) / \mathrm{d} \mathbf{q})$, in modern language. Using the notations of the present article, it reads as follows.

Problem $\mathcal{P}_{5}$. Find $\mathbf{q} \in M M A\left([0, T] ; \mathbb{R}^{2}\right)$ and $\mathbf{r}, \tilde{\mathbf{r}} \in \mathcal{M}\left([0, T] ; \mathbb{R}^{2}\right)$ such that:

- $\mathbf{q}(0)=\mathbf{q}_{0}, \quad \dot{\mathbf{q}}(0)=\mathbf{v}_{0} \quad$ (initial condition),

- $\mathbf{M}(\mathbf{q}) \cdot \frac{\mathrm{D} \dot{\mathbf{q}}}{\mathrm{D} t}=\mathbf{f}(\mathbf{q})+\mathbf{r} \quad$ (equation of motion, in the sense of distributions),

- $\tilde{\mathbf{r}}={ }^{\mathrm{t}} \mathbf{l}^{-1}(\mathbf{q}, \mathbf{x}) \cdot \mathbf{r} \quad$ (expression of real world reaction force)

- $\theta-\theta_{0} \geq 0, \quad \tilde{r}_{y} \geq 0, \quad\left(\theta-\theta_{0}\right) \tilde{r}_{y} \equiv 0 \quad$ (unilateral contact)

- $\forall \hat{v} \in C^{0}([0, T] ; \mathbb{R}), \quad \int_{[0, T]} \tilde{r}_{x}\left(\hat{v}-\dot{x}+l \sin \theta_{0} \dot{\theta}\right)+\mu \tilde{r}_{y}\left(|\hat{v}|-\left|\dot{x}-l \sin \theta_{0} \dot{\theta}\right|\right) \geq 0$

(Coulomb friction)

- $\theta=\theta_{0} \quad \Rightarrow \quad \dot{\theta}^{+}=0 \quad$ (completely inelastic impact law)

In [26], Stewart proved the existence of a solution for problem $\mathcal{P}_{5}$ (and more generally for any such problem in the plane with one contact point and the completely inelastic impact law). He also proved that there exists a dissipative solution (one for which the sum of kinetic and potential energy decreases with time). This article was intended as a contribution to a recurring controversy during the whole 
twentieth century about the frictional unilateral rigid body dynamics as formulated in problem $\mathcal{P}_{5}$. Indeed, the formulation of problem $\mathcal{P}_{5}$ was charged to raise many inconsistencies. Among them, there were Painlevé's paradoxes (non-uniqueness and even suspicion of non-existence of solution) and Kane's paradox (the friction law can be responsible for the kinetic energy to increase during an impact).

\section{Painlevé's paradox.}

- Non-existence of solution. We consider the following initial conditions at $t=0$ :

$$
\begin{aligned}
x(0) & =0, & \dot{x}(0) & =V_{0}>0, \\
\theta(0) & =\theta_{0}, & \dot{\theta}(0) & =0,
\end{aligned}
$$

and the following loading conditions for $t \geq 0$ :

$$
f_{x}(\mathbf{q})=0, \quad f_{\theta}=C_{0}<0 .
$$

Any smooth solution satisfies

$$
J \ddot{\theta}=C_{0}+l \tilde{r}_{y}\left(\cos \theta_{0}+\mu \sin \theta_{0}\right),
$$

in a right-neighbourhood of the initial instant. Since $\mu>-1 / \tan \theta_{0}$ and $\tilde{r}_{y} \geq 0$, one necessarily has $\ddot{\theta}<0$ which is incompatible with the contact condition. Therefore, no smooth solution exists for the dynamical evolution problem. Painlevé had considered this fact as possible non-existence of solution for the frictional unilateral dynamics. Lecornu [12] then pointed out that existence of solution could be recovered by allowing an impact (velocity jump and associated Dirac mass of the reaction measure) at initial instant $t=0$. This impact, called 'tangential impact' or 'frictional catastrophe' by Moreau [18], must have vanishing right-velocity $\dot{x}^{+}(0)=0, \dot{\theta}^{+}(0)=0$ and the subsequent motion is immobility. In the case of a system where the $y$-coordinate of point $G$ is not constrained to have a constant value, an example where such a 'frictional catastrophe' must occur after a phase of smooth sliding can easily be designed. Therefore, the existence result of Stewart in [26] was validating the suggestion of Moreau that 'frictional catastrophes' were a possible way to recover existence of solution in a systematic manner, invalidating the original objection of Painlevé. Let us point out, once again, that the existence result of Stewart was restricted to the situation of a plane problem, with only one material contact point, and the completely inelastic impact law.

- Non-uniqueness of solution. We consider the following initial conditions at $t=0$ in problem $\mathcal{P}_{5}$ :

$$
\begin{array}{ll}
x(0)=0, & \dot{x}(0)=V_{0}>0, \\
\theta(0)=\theta_{0}, & \dot{\theta}(0)=0,
\end{array}
$$

and the following loading conditions for $t \geq 0$ :

$$
f_{x}(\mathbf{q}) \equiv 0, \quad f_{\theta} \equiv 0 .
$$

Then, picking an arbitrary $t_{0} \geq 0$, the motion with grazing contact for $t \in\left[0, t_{0}\right]$ and immobility for $t \geq t_{0}$ :

$$
\begin{aligned}
& (x(t), \theta(t))=\left(V_{0} t, \theta_{0}\right), \\
& \text { for } t \in\left[0, t_{0}\right] \text {, } \\
& (x(t), \theta(t))=\left(V_{0} t_{0}, \theta_{0}\right), \\
& \text { for } t>t_{0} \text {, }
\end{aligned}
$$

are all a solution for problem $\mathcal{P}_{5}$ in the case where $\mu>-1 / \tan \theta_{0}$. This non-uniqueness was judged as paradoxical by Painlevé and acceptable by Moreau (in a context of numerical modelling of granular materials).

2. Kane's paradox. When using the paradigm of Coulomb's friction law applying to reaction forces of the real world as in problem $\mathcal{P}_{5}$, there is no 'built-in' guarantee of dissipativity of the friction law, especially during collisions. In [10], Kane gave a striking example for the dynamics of a double pendulum where a frictional collision leads to an increase of energy in the system (this 
example can also be found in [11], problem 14.6). For the particular situation he considered (plane problem, one contact point, and completely inelastic impact law situation), Stewart showed that there always exists a solution that does not exhibit this pathology. The example of Kane, shows that Stewart's dissipativity result could not be extended to the case of a restitution coefficient of 0.5 .

Now, let us formulate the frictional unilateral dynamic of this rigid bar along the principle advocated in this paper. Since the unilateral constraint reads as:

$$
\varphi(\theta)=\theta-\theta_{0} \geq 0
$$

the normal component of the (generalized) reaction force is $r_{\theta}$ and the tangential component is $r_{x}$ (according to the definition given in section 3.1), so that the contact condition should be written as: parametrization read as:

$$
\theta-\theta_{0} \geq 0, \quad r_{\theta} \geq 0, \quad\left(\theta-\theta_{0}\right) r_{\theta} \equiv 0,
$$

and the friction cone should be postulated under the form:

$$
r_{x} \in r_{\theta} \mathcal{C}_{\mathbf{q}}^{*}
$$

where $\mathcal{C}_{\mathbf{q}}^{*}$ is a nonempty closed interval, according to the content of section 3.2. Implementing the procedure described in section 6.1 to construct $\mathcal{C}_{\mathbf{q}}^{*}$ based on the paradigm of Coulomb friction applying on real word reaction forces, we obtain:

$$
\begin{array}{ll}
\mathcal{C}_{\mathbf{q}}^{*}=\left[-\frac{\mu}{l\left(\cos \theta_{0}+\mu \sin \theta_{0}\right)}, \frac{\mu}{l\left(\cos \theta_{0}-\mu \sin \theta_{0}\right)}\right], & \text { if } \mu<-1 / \tan \theta_{0}, \\
\left.\left.\mathcal{C}_{\mathbf{q}}^{*}=\right]-\infty, \frac{\mu}{l\left(\cos \theta_{0}-\mu \sin \theta_{0}\right)}\right], & \text { if } \mu \geq-1 / \tan \theta_{0} .
\end{array}
$$

Note that the procedure described in section 6.1 produces a bounded friction $\operatorname{set} \mathcal{C}_{\mathbf{q}}^{*}$ for small friction coefficient $\left(\mu<-1 / \tan \theta_{0}\right)$, whereas $\mathcal{C}_{\mathbf{q}}^{*}$ turns out to be unbounded in the case of a larger friction coefficient.

Making that choice for the friction set $\mathcal{C}_{\mathbf{q}}^{*}$, our formulation of the dynamics (problem $\mathcal{P}_{3}^{\prime}$ ) reduces, in case of the completely inelastic impact law to the following.

Problem $\mathcal{P}_{3}^{\prime}$. Find $\mathbf{q} \in M M A\left([0, T] ; \mathbb{R}^{2}\right)$ and $\mathbf{r} \in \mathcal{M}\left([0, T] ; \mathbb{R}^{2}\right)$ such that:

- $\mathbf{q}(0)=\mathbf{q}_{0}, \quad \dot{\mathbf{q}}(0)=\mathbf{v}_{0} \quad$ (initial condition),

- $\mathbf{M}(\mathbf{q}) \cdot \frac{\mathrm{D} \dot{\mathbf{q}}}{\mathrm{D} t}=\mathbf{f}(\mathbf{q})+\mathbf{r} \quad$ (equation of motion, in the sense of distributions),

- $\theta-\theta_{0} \geq 0, \quad r_{\theta} \geq 0, \quad\left(\theta-\theta_{0}\right) r_{\theta} \equiv 0 \quad$ (unilateral contact)

- $\forall \hat{v}_{x} \in C^{0}([0, T] ; \mathbb{R}), \quad \int_{0}^{T} r_{x}\left(\hat{v}_{x}-\dot{x}^{+}\right)+r_{\theta}\left[S_{\mathcal{C}_{\mathbf{q}}^{*}}\left(-\hat{v}_{x}\right)-S_{\mathcal{C}_{\mathbf{q}}^{*}}\left(-\dot{x}^{+}\right)\right] \geq 0$

(generalized friction law),

- $\theta=\theta_{0} \quad \Rightarrow \quad \dot{\theta}^{+}=0 \quad$ (completely inelastic impact law).

Let us recall that in the case where $\mathcal{C}^{*}=[a, b]$ with $a \leq 0$ and $b \geq 0$, then $D\left(S_{\mathcal{C}^{*}}\right)=\mathbb{R}$ and:

$$
S_{\mathcal{C}^{*}}(\hat{v})=b \max \{\hat{v}, 0\}-a \max \{-\hat{v}, 0\} .
$$

In the case where $\left.\left.\mathcal{C}^{*}=\right]-\infty, b\right]$ with $b \geq 0$, then $D\left(S_{\mathcal{C}^{*}}\right)=\mathbb{R}^{+}$and:

$$
\forall \hat{v} \in \mathbb{R}^{+}, \quad S_{\mathcal{C}^{*}}(\hat{v})=b \hat{v} .
$$

Based on this, the following facts are readily checked.

- If $\mu<-1 / \tan \theta_{0}$, then $(\mathbf{q}, \mathbf{r}) \in M M A\left([0, T] ; \mathbb{R}^{2}\right) \times \mathcal{M}\left([0, T] ; \mathbb{R}^{2}\right)$ is a solution of problem $\mathcal{P}_{3}^{\prime}$ if and only if it is a solution of problem $\mathcal{P}_{5}$. 
- If $\mu<-1 / \tan \theta_{0}$ and the function $\mathbf{f}$ is an analytic function of $t, \mathbf{q}, \dot{\mathbf{q}}$, then the analysis in [5] can readily be transposed to yield the existence of a unique solution of problem $\mathcal{P}_{3}^{\prime}$. The same is therefore true for problem $\mathcal{P}_{5}$ in that case. This is an improvement of Stewart's result in that case, since he had obtained the existence of a solution with no information on its uniqueness.

- If $\mu \geq-1 / \tan \theta_{0}$, then any solution $(\mathbf{q}, \mathbf{r}) \in M M A\left([0, T] ; \mathbb{R}^{2}\right) \times \mathcal{M}\left([0, T] ; \mathbb{R}^{2}\right)$ of problem $\mathcal{P}_{3}^{\prime}$ is a solution of problem $\mathcal{P}_{5}$. In addition, we can make the following remarks.

- Considering problem $\mathcal{P}_{3}^{\prime}$ with the initial condition:

$$
\begin{array}{ll}
x(0)=0, & \dot{x}(0)=V_{0}>0, \\
\theta(0)=\theta_{0}, & \dot{\theta}(0)=0,
\end{array}
$$

and the following loading conditions for $t \geq 0$ :

$$
f_{x}(\mathbf{q})=0, \quad f_{\theta}=C_{0}<0,
$$

it is readily checked that the motion with 'tangential impact' at initial time followed by immobility provides a solution for problem $\mathcal{P}_{3}^{\prime}$ in this case. So, the 'frictional catastrophe' of Moreau [18] can also happen in our setting. But, they can only happen in the case of an unbounded friction set. Hence, a qualitative conclusion about the dynamics can be drawn, based on the static analysis described in section 6.1 to construct the friction sets based on the paradigm of Coulomb law applying to real world reaction force: if all the friction sets are bounded, no 'frictional catastrophe' is to be expected.

- Consider now problem $\mathcal{P}_{3}^{\prime}$ with the initial condition:

$$
\begin{array}{ll}
x(0)=0, & \dot{x}(0)=V_{0}>0, \\
\theta(0)=\theta_{0}, & \dot{\theta}(0)=0,
\end{array}
$$

and the following loading conditions for $t \geq 0$ :

$$
f_{x}(\mathbf{q})=0, \quad f_{\theta}=0 .
$$

Multiplying the first component of the equation of motion $\ddot{\theta}=r_{\theta}$ by $\dot{\theta}^{+}+\dot{\theta}^{-}$, and integrating, it is readily seen that $\left(\dot{\theta}^{+}\right)^{2}$ is nonincreasing. Therefore, one obtains that $\dot{\theta} \equiv 0$ and therefore $r_{\theta} \equiv 0$. But, by the friction law, we also have $r_{x} \equiv 0$. Hence, grazing contact $(x(t), \theta(t))=\left(V_{0} t, \theta_{0}\right)$ is the unique solution of problem $\mathcal{P}_{3}^{\prime}$. We recall that problem $\mathcal{P}_{5}$ had infinitely many solutions in that case, with immobility possibly arising after a 'frictional catastrophe' at any instant $t_{0}$. Hence, the formulation as problem $\mathcal{P}_{3}^{\prime}$ fixes the indeterminacy of the Painlevé example by 'chosing' one solution among all. Of course, a definitive proof that the new formulation fixes the Painlevé paradox requires the proof that there exists a unique solution in general for analytic data, even in the case of unbounded friction set $\mathcal{C}_{\mathbf{q}}^{*}$ $\left(\mu \geq-1 / \tan \theta_{0}\right)$, but the proof is postpone to future work. However, the example under scrutiny makes plausible the validity of such a result.

Summing up, we have compared in the case of this simple example, the evolution problems obtained by respectively:

- writing the Coulomb friction law applying on real world reaction forces (problem $\mathcal{P}_{5}$ ),

- writing a friction law in terms of the generalized reaction forces with a friction set postulated on the basis of the paradigm of the Coulomb friction law applying on real world reaction forces (problem $\mathcal{P}_{3}^{\prime}$ ).

Although this simple example is embodied in the particular circumstance where the real world reaction forces and the generalized reaction force are linked through an invertible relation, the two evolution problems $\mathcal{P}_{5}$ and $\mathcal{P}_{3}^{\prime}$ are not equivalent. The reason is that the normal component $r_{\mathrm{n}}=r_{\theta}$ of the generalized reaction is required to be nonnegative in problem $\mathcal{P}_{5}$ whereas generalized reaction forces with negative normal component can arise in problem $\mathcal{P}_{3}^{\prime}$ whenever $\mu>-1 / \tan \theta_{0}$.

The two evolution problems were found equivalent in the case where $\mu<-1 / \tan \theta_{0}$. In the case where $\mu \geq-1 / \tan \theta_{0}$, the first approach leads to an evolution problem which may have multiple 
solutions whereas the indeterminacy is broken in the second approach which selects one of the multiple solutions. Nonetheless, the first approach is the one which is usually considered and is often stated as more physical, as it is easier to get a physical intuition of real world reaction forces than of generalized reaction forces. In this philosophy, it is claimed that the indeterminacy observed in the case $\mu \geq-1 / \tan \theta_{0}$ is part of the physics and should be accepted. This indeterminacy is fixed when the formulation is made in terms of generalized reaction force (problem $\mathcal{P}_{3}^{\prime}$ ). Our opinion is that the origin of the indeterminacy in problem $\mathcal{P}_{5}$ is that the formulation has not been made on the basis of the Lagrange's philosophy of virtual power principle which requires that the friction law should be expressed in terms of generalized reaction force and not in terms of real world reaction force. Sticking to this principle inevitably leads to a formulation along the lines of problem $\mathcal{P}_{3}^{\prime}$ which seems to fix the indeterminacy (a definitive proof of that will be a proof of existence and uniqueness for problem $\mathcal{P}_{4}^{\prime}$ which will be tackled in future work).

More importantly, the example of Kane is very similar to this one (plane dynamical problem for a double pendulum with only one material contact point), and the problem can be given a formulation along problem $\mathcal{P}_{5}$ here (except that the impact law involves a coefficient of restitution of 0.5 instead of 0 ). Then, the analysis of Kane shows that there are pre-collision velocity conditions that necessarily produce a collision during which there is an increase of the kinetic energy of the system. This is an important drawback of the formulation in terms of Coulomb friction law applying on real world reaction forces: energetic consistency is not guaranteed. The analysis of Stewart in [26] shows that this pathology is precluded, at least for the solution built by means of his numerical method, for the plane problem with only one contact material point and the completely inelastic impact law. There is no guarantee of energetic consistency otherwise when the problem is formulated in terms of the Coulomb friction law applying on real world reaction forces. On the contrary, energetic consistency is guaranteed in the most general case when the problem is formulated along the lines of problem $\mathcal{P}_{4}^{\prime}$, thanks to proposition 5.1.

In any case, it should be pointed out that this example (and all the one material contact point problem that are often considered in such a discussion) is very specific because the mapping $\mathbf{l}(\mathbf{q}, \mathbf{x})$ that allows to compute the generalized reaction force in terms of the real world reaction force is one-to-one in this type of example, whereas the mapping $\mathbf{l}(\mathbf{q}, \mathbf{x})$ is not invertible in general. In the next section, we shall consider examples in which the mapping $\mathbf{l}(\mathbf{q}, \mathbf{x})$ is not invertible. We shall see that the approach based on the paradigm of Coulomb friction applying on reaction forces of the real world (along problem $\mathcal{P}_{5}$ ) is much less amenable than in the simple situation that was examined in this subsection.

\subsection{Further examples}

In this section, two simple systems will be considered. They have in common that the mapping

$$
\left(\tilde{\mathbf{r}}_{i}\right) \mapsto \mathbf{r}=\sum_{i}{ }^{\mathrm{t}} \mathbf{l}\left(\mathbf{q}, \mathbf{x}_{i}\right) \cdot \tilde{\mathbf{r}}_{i}
$$

is not invertible, unlike the example considered in the previous section. The objective is to compare the evolution problem obtained using the paradigm of Coulomb friction applying on the real world reaction forces with the problem $\mathcal{P}_{4}^{\prime}$.

Let $A B$ be a rigid bar with center of mass $G$ evolving in the plane. The situation where the location of $B$ is prescribed and invariable, the bar being only able to rotate around $B$ is called 'system 1 ' in the sequel. The situation where $B$ can move freely is called 'system 2 ' in the sequel. System 2 can be parametrized by using the coordinates $(x, y)$ of $G$ and the angle $\theta$ from the initial direction of the bar to the current direction. System 2 has therefore three degrees of freedom. System 1 can be parametrized by $\theta$ only and has therefore one degree of freedom. Generalized forces in system 1 have only one component and generalized forces in system 2 have three components: the $x$ and $y$ components of the total force and the torque evaluated at $G$. We also consider a concave nonstraight rigid obstacle as represented in figure 2 and wish to analyze a motion of the bar (system 1 or 2) above the obstacle with dry friction taken into account when contact is active. 

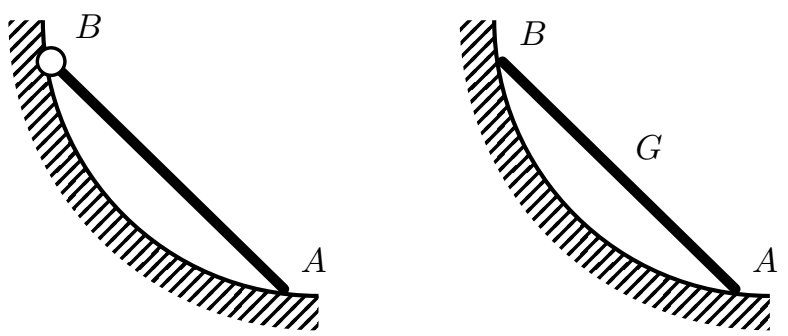

Figure 2. Two simple discrete mechanical systems with one (system 1, left) and three (system 2, right) d.o.f.

We first examine system 1 . This system is naturally parametrized by use of the angle $\theta$ made by the bar with its own direction when it is in contact with the obstacle. The generalized reaction $r_{\theta}$ force has only one component and is to be interpreted in terms of a torque at point $B$ exerted by the reaction force. The corresponding evolution problem $\mathcal{P}_{3}^{\prime}$ is trivial. It involves no friction set since system 1 , having only one degree of freedom, is formally identical to the case of a point particle moving along a line on one side of a given obstacle. It reads as follows.

Problem $\mathcal{P}_{3}^{\prime}$. Find $\theta \in M M A([0, T] ; \mathbb{R})$ and $r_{\theta} \in \mathcal{M}([0, T] ; \mathbb{R})$ such that:

- $\theta(0)=\theta_{0}, \quad \dot{\theta}(0)=\omega_{0} \quad$ (initial condition),

- $J \ddot{\theta}=f_{\theta}+r_{\theta} \quad$ (equation of motion, in the sense of distributions),

- $\theta \geq 0, \quad r_{\theta} \geq 0, \quad \theta r_{\theta} \equiv 0 \quad$ (unilateral contact)

- $\theta=0 \quad \Rightarrow \quad \dot{\theta}^{+}=-e \dot{\theta}^{-} \quad$ (impact law).

Here, $J$ denotes inertia momentum of the bar around point $B, f_{\theta}$ (which may depend on $(t ; \theta, \dot{\theta})$ ) the given external force, and $e \in[0,1]$ a given restitution coefficient. With the usual paradigm of the Coulomb law applying to real world reaction forces, one would get the following evolution problem. If $f_{\theta}$ is an analytic function of $t$ only (to simplify), then problem $\mathcal{P}_{3}^{\prime}$ admits a unique solution, thanks to [1].

Problem $\mathcal{P}_{6}$. Find $\theta \in M M A([0, T] ; \mathbb{R}), r_{\theta} \in \mathcal{M}([0, T] ; \mathbb{R})$ and $\left(\tilde{r}_{\mathrm{n}}, \tilde{r}_{\mathrm{t}}\right) \in \mathcal{M}\left([0, T] ; \mathbb{R}^{2}\right)$ such that:

- $\theta(0)=\theta_{0}, \quad \dot{\theta}(0)=\omega_{0} \quad$ (initial condition),

- $J \ddot{\theta}=f_{\theta}+r_{\theta} \quad$ (equation of motion, in the sense of distributions),

- $r_{\theta}=\tilde{r}_{\mathrm{t}} \cos \tilde{\theta}_{0}+\tilde{r}_{\mathrm{n}} \sin \tilde{\theta}_{0}$.

- $\theta \geq 0, \quad \tilde{r}_{\mathrm{n}} \geq 0, \quad \theta \tilde{r}_{\mathrm{n}} \equiv 0 \quad$ (unilateral contact)

- $\forall \hat{v} \in C^{0}([0, T] ; \mathbb{R}), \quad \int_{[0, T]} \tilde{r}_{\mathrm{t}}\left(\hat{v}-l \sin \tilde{\theta}_{0} \dot{\theta}\right)+\mu \tilde{r}_{\mathrm{n}}\left(|\hat{v}|-\left|l \sin \tilde{\theta}_{0} \dot{\theta}\right|\right) \geq 0$

(Coulomb friction)

- $\theta=0 \Rightarrow \dot{\theta}^{+}=-e \dot{\theta}^{-} \quad$ (impact law).

Here, $\tilde{\theta}_{0}$ denotes the angle between the tangent to the obstacle and the direction of the bar when contact is active, and $\tilde{r}_{\mathrm{n}}, \tilde{r}_{\mathrm{t}}$ respectively the normal and tangential component of the real world reaction force. In the case where $\mu>\tan \tilde{\theta}_{0}>0, \theta_{0}=0, \omega_{0}=0$ and $f_{\theta} \equiv C_{0}>0$, then the following motion:

$$
\begin{aligned}
& \theta(t)=0, \\
& \tilde{r}_{\mathrm{n}}(t)=\frac{C_{0}}{\mu \cos \tilde{\theta}_{0}-\sin \tilde{\theta}_{0}} \\
& \tilde{r}_{\mathrm{t}}(t)=-\frac{\mu C_{0}}{\mu \cos \tilde{\theta}_{0}-\sin \tilde{\theta}_{0}}, \quad \text { if } t \in\left[0, t_{0}[,\right. \\
& \theta(t)=C_{0}\left(t-t_{0}\right)^{2} /(2 J), \quad \tilde{r}_{\mathrm{n}}(t)=0, \\
& \tilde{r}_{\mathrm{t}}(t)=0, \\
& \text { if } \left.t \in] t_{0}, T\right] \text {, }
\end{aligned}
$$

defines a solution of problem $\mathcal{P}_{6}$, for all $t_{0} \in[0, T]$. In this example, the bar is initially at rest in contact with the obstacle and a constant positive external torque is applied on the bar. Any solution such 
that the bar is stuck against the obstacle during an arbitrary time $t_{0}$ and then is accelerating away, is possible.

The analysis of system 2 leads to similar conclusions. When points $A$ and $B$ are in contact with the obstacle, the real world reaction forces have four independant components whereas system 2 has only three degrees of freedom. Therefore, the real world forces cannot be recovered from the generalized reaction force. When the evolution problems that governs the dynamics is written using the paradigm of the Coulomb law applying to real world reaction forces, one gets the same kind of indetermination with possible wedged equilibrium under constant forces, that suddenly breaks at an arbitrary instant. On the contrary, if a formulation along the lines of problem $\mathcal{P}_{4}^{\prime}$ is adopted, with a constant external force pulling away the bar from the obstacle, then it is possible to follow the same line of reasoning as in subsection 6.2 to prove that the generalized reaction force must vanish identically and no episode of wedged equilibrium is therefore possible.

\subsection{Discussion and conclusion}

The supporters of the use of the paradigm of Coulomb friction applying on real world reaction forces sometimes argue that the multiple solutions displayed in the previous section should be accepted since it is observed that real bars can either be wedged or not in experiments. However, it can be retorted that solutions displaying a broken wedged equilibrium at an arbitrary instant are not so physical. Moreover, solutions displaying increase of kinetic energy during an impact as in Kane's example are very questionable from a physical point of view. Finally, from a practical point of view, to show that a given motion $\mathbf{q}(t)$ solves the evolution problem based on the real world reaction forces, one has to construct some associated real world reaction forces $\tilde{r}$ and, likewise, to discard a motion as a possible solution, one has to prove that an associated real world reaction forces $\tilde{r}$ does not exist. This is not amenable in practice and is going to preclude almost all further theoretical investigations of the evolution problem based on real world reaction forces, except for very simple systems. The only case where such a theoretical investigation is actually possible is the very specific case of one material contact point since in this case the real world reaction force can be calculated in terms of the generalized reaction force. This is precisely the case in which Stewart [26] performed his analysis.

Our opinion is that all these difficulties originate in the use of reaction forces (the real world reaction forces) that are inadequate to the geometric modelling of the mechanical system under scrutiny. It has been known since Lagrange that the appropriate representation of forces in any mechanical theory is the one that arises from duality with velocities in the virtual power approach. This point of view has been progressively adopted in the mechanics of continua during the twentieth century. For example, it is now fully recognized that the representation of internal forces in a continuum in terms of the Cauchy stress field is appropriate for a classical deformable continuum, but it is not appropriate for a rigid body (in which it is indeterminate) and it is not appropriate either in a Cosserat medium. For a collection of rigid bodies, the appropriate representation of forces are generalized forces and not real world forces. One has to accept that real world reaction forces remain generally indeterminate and that the friction law must be written in terms of the generalized reaction forces. Sticking to real world reaction forces is at the origin of both indeterminacies and energy inconsistencies (Painlevé and Kane paradoxes) in multibody frictional unilateral dynamics. Besides, the original Coulomb law based on his own experiments was formulated in terms of total tangential force and total normal force, which are generalized forces and not real world forces.

For systems of point particles, generalized forces and real world force coincide and the two points of view are identical. This is not true for arbitrary rigid bodies. In the case of the bar in systems 1 and 2 represented on figure 2 , with friction set $\mathcal{C}_{\mathbf{q}}^{*}$ postulated by use of the systematic method described in section 6.1 , both points of view will give the same prediction of motion for small friction coefficient (more precisely for friction coefficient that are sufficiently small to render wedging impossible). For larger friction coefficients, the real world forces point of view has an indeterminacy (wedging or non wedging are both possible). The point of view of generalized forces discards wedging and thus breaks the indeterminacy. Supporters of the point of view of real world forces may feel frustrated since wedging can be encountered in practice. This is only an inadequacy of the rigid bar model to discuss the wedging 
phenomenon which is fundamentally governed by the necessary deformability of a real bar. A refined model must be introduced, for example a model in which the two end points are connected by a spring. In this refined model, the real world forces and the generalized forces coincide and it is now possible to discuss the eventuality of the wedging phenomenon.

In the context of numerical modelling where the objective is to produce realistic examples of motion of a system of rigid bodies, the paradigm of Coulomb friction applying on real world reaction forces has been successfully used to design numerical methods. However, this point of view contradicts the basic principles of modern mechanics and does not enable one to write down a consistent evolution problem. This is the origin of the paradoxes of Painlevé and Kane. Our opinion is that the evolution problem has to be formulated from the beginning in terms of the generalized reaction force (this is problem $\mathcal{P}_{4}^{\prime}$ in this paper), and that the paradigm of Coulomb friction applying on real world reaction forces can only be invoked in a second step as a heuristic (but systematic) way to identify the friction sets entering problem $\mathcal{P}_{4}^{\prime}$. We have proved that dissipativity of solution is built-in within this new formulation which fixes the paradox of Kane. We also have proved that this new formulation fixes some indeterminacies of the Painlevé paradox, making it very plausible that this new formulation is free of Painlevé paradox. A definitive answer would be a proof of existence and uniqueness of solutions for problem $\mathcal{P}_{4}^{\prime}$ under analyticity hypotheses of the data as it has already been proved for frictionless unilateral multibody dynamics in [1].

\section{References}

[1] P. Ballard, The dynamics of discrete mechanical systems with perfect unilateral constraints. Archive for Rational Mechanics and Analysis 154 (2000), 199-274.

[2] P. Ballard, Formulation and well-posedness of the dynamics of rigid-body systems with perfect unilateral constraints. Philosophical Transactions of the Royal Society, A 359 (2001), 2327-2346.

[3] P. Ballard, Dynamics of rigid bodies systems with unilateral or frictional constraints. In: D.Y. Gao, R. Ogden (eds.) Advances in mechanics and mathematics, vol. 1 (2002), chap. 1, pp. 3-88. Kluwer Academic Publishers.

[4] H. Béghin, Sur certains problèmes de frottement. Nouvelles Annales de Mathématiques 5ème série 2 (1923), 305-312.

[5] A. Charles and P. Ballard, Existence and uniqueness of solutions to dynamical unilateral contact problems with Coulomb friction: the case of a collection of points. Mathematical Modelling and Numerical Analysis 48(1) (2014), 1-25.

[6] A. Coulomb, Théorie des machines simples. Bachelier, Paris (1821).

[7] C. Glocker, Energetic consistency conditions for standard impacts, part $i$ : Newton-type inequality impact laws and Kane's example. Multibody System Dynamics 29 (2013), 77-117.

[8] C. Godbillon, Géométrie Différentielle et Mécanique Analytique. Hermann, Paris (1969).

[9] M. Jean, The non-smooth contact dynamics method. Computer Methods in Applied Mechanics and Engineering 177 (1999), 235-257.

[10] T.R. Kane, A dynamics puzzle. Stanford Mechanics Alumni Club Newsletter p. 6 (1983).

[11] T.R. Kane and D.A. Levinson, Dynamics : theory and applications. McGrawHill, New-York (1985).

[12] L. Lecornu, Sur la loi de Coulomb. Comptes Rendus De l'académie Des Sciences (Paris) 140 (1905), $847-848$.

[13] R. Michałovsky and Z. Mróz, Associated and non-associated sliding rules in contact friction problems. Archives of Mechanics 30(3) (1978), 259-276.

[14] M.D.P. Monteiro Marques, Differential inclusions in nonsmooth mechanical problems - shocks and dry friction, Progress in nonlinear differential equations and their applications, vol. 9. Birkhäuser, Basel, Boston, Berlin (1993).

[15] J.J. Moreau, Standard inelastic shocks and the dynamics of unilateral constraints. In: G. Del Piero, F. Maceri (eds.) Unilateral problems in structural analysis, pp. 173-221. Springer-Verlag, Wien, New-York (1983). 
[16] J.J. Moreau, Une formulation du contact à frottement sec ; application au calcul numérique. Comptes Rendus De l'académie Des Sciences (Paris), série II, 302 (1986), 799-801.

[17] J.J. Moreau, Bounded variation in time. In: J.J. Moreau, P.D. Panangiatopoulos, G. Strang (eds.) Topics in non-smooth mechanics, chap. 1, pp. 1-74. Birkhäuser Verlag, Basel-Boston-Berlin (1988).

[18] J.J. Moreau, Unilateral contact and dry friction in finite freedom dynamics. In: J.J. Moreau, P.D. Panagiotopoulos (eds.) Non-smooth mechanics and applications, pp. 1-82, Springer-Verlag, Wien, New-York (1988).

[19] P. Painlevé, Sur les lois du frottement de glissement. Comptes Rendus De l'académie Des Sciences (Paris) 121 (1895), 112-115.

[20] L. Paoli, Time-stepping approximation of rigid-body dynamics with perfect unilateral constraints. I and II. Archive for Rational Mechanics and Analysis 198 (2010), 457-503 and 505-568.

[21] L. Paoli, A proximal-like algorithm for vibro-impact problems with a non smooth set of constraints. Journal of Differential Equations 250 (2011), 476-514.

[22] S. Pasquero, Nonideal unilateral constraints in impulsive mechanics: a geometric approach. Journal of Mathematical Physics 49(4) (2008).

[23] D. Percivale, Uniqueness in the elastic bounce problem, i. Journal of Differential Equations 2(2) (1985), 206-215.

[24] D. Percivale, Uniqueness in the elastic bounce problem, ii. Journal of Differential Equations 90, 304-315 (1991)

[25] M. Schatzman, A class of nonlinear differential equations of second order in time. Nonlinear Analysis, Theory, Methods and Applications 2(2) (1978), 355-373.

[26] D.E. Stewart, Convergence of a time-stepping scheme for rigid-body dynamics and resolution of painlevé's problem. Archive for Rational Mechanics and Analysis 145 (1998), 215-260.

[27] D.E. Stewart, Rigid-Body Dynamics with Friction and Impact. SIAM Review 42(1) (2000), 3-39.

Alexandre Charles

Laboratoire de Mécanique et d'Acoustique, CNRS

31, Chemin Joseph Aiguier

13402 Marseille Cedex 20, France

Patrick Ballard

Laboratoire de Mécanique et d'Acoustique, CNRS

31, Chemin Joseph Aiguier

13402 Marseille Cedex 20, France

e-mail: ballard@lma.cnrs-mrs.fr 